hep-th/0207097

July, 2002

\title{
Kronecker foliation, D1-branes and
}

\section{Morita equivalence of Noncommutative two-tori}

\author{
Hiroshige Kajiura [ \\ Graduate School of Mathematical Sciences, University of Tokyo \\ 3-8-1 Komaba, Tokyo, 153-8914 Japan
}

\begin{abstract}
It is known that the physics of open strings on a D2-brane on a two-torus is realized from the viewpoint of deformation quantization in the Seiberg-Witten limit. We study its T-dual theory, i.e. D1-brane physics on two-tori. Such theory is described by Kronecker foliation. The algebra of open strings on the D1-brane is then identified with the crossed product representation of a noncommutative two-torus. The Morita equivalence of noncommutative two-tori is also realized geometrically along this line. As an application, Heisenberg modules and the tensor product between them are discussed from these geometric viewpoints. We show they are related to the homological mirror symmetry of two-tori.
\end{abstract}

*e-mail address: kuzzy@ms.u-tokyo.ac.jp 


\section{Contents}

\begin{tabular}{lll}
\hline Introduction & 1
\end{tabular}

\begin{tabular}{|lll}
\hline Kronecker foliation and Morita equivalence & $\mathbf{3}$
\end{tabular}

$2.1 \quad$ Kronecker foliation and noncommutative torus . . . . . . . . . . . . . . . . 3

2.2 Kronecker foliation and Morita equivalence . . . . . . . . . . . . . . 5

$\begin{array}{lll}3 \text { Open string ending on D1-brang } & 6\end{array}$

$3.1(p, q)=(0,1)$ cast . . . . . . . . . . . . . . . . . . . . . 7

3.2 General $(p, q)$ case $\ldots \ldots \ldots \ldots$

4 The Seiberg-Witten limit and the Kronecker foliation limit 9

$\begin{array}{lll}5 \text { Application } & 10\end{array}$

5.1 Projective modules from the Kronecker foliation . . . . . . . . . . . . . . . 10

5.2 Relation to the homological mirror symmetry . . . . . . . . . . . . . . . . 15

6 Conclusions and Discussions 23

\section{Introduction}

Noncommutative geometry is directly related to the physics of open strings [1]-[7]. A noncommutative torus is an ideal example of noncommutative algebras. It is known that noncommutative tori have a beautiful symmetry, the Morita equivalence [8]-11]. The Morita equivalence is an important concept also for physics. It is the equivalence of (noncommutative) algebras which have the isomorphic category of projective modules $\square$. Here, in the spirit of the noncommutative geometry, a noncommutative algebra is regarded as the space of functions on a 'noncommutative space'. The projective modules over a noncommutative algebra are then vector bundles, i.e. D-branes over the noncommutative space. Therefore one can say physically that the Morita equivalent algebras are the noncommutative target spaces on which the same set of D-branes exists. Furthermore, if one has a background independent field theory of open strings, each D-brane should correspond to each classical solution of the field theory. Actually, for the effective field theory of open strings on (two-)tori, it is shown that each classical solution corresponds to each brane 12, 13] in the context of tachyon condensation [14]. Thus, the investigation of noncommutative algebras and their Morita equivalence is an important subject which is relevant to the nonperturbative effects and furthermore the background independence

\footnotetext{
${ }^{1}$ There exists another but equivalent (more explicit) definition of the Morita equivalence, which is stated in Subsection 5.1.
} 
of the field theory of open strings. In addition, noncommutative expressions have the advantage that projective modules over a noncommutative space can uniformly describe D-branes including those which cannot be described by vector bundles. For instance, D0-brane on a two-torus with no D2-brane cannot be expressed in terms of a vector bundle over the two-torus, but can be expressed in terms of a projective module over a noncommutative-torus.

Noncommutative tori are represented in several ways; deformation quantization 15, irrational rotation algebra [16, 17, and so on. For simplicity, let us concentrate on twodimensional tori. Open string theory on a D2-brane can be realized from the viewpoint of the deformation quantization [5, 6], so it is described by a noncommutative two-torus. Morita equivalent noncommutative two-tori are then generated by group $S L(2, \mathbb{Z})$. It is known to be related to T-duality group, so the Morita equivalent noncommutative two-tori can be regarded as the algebras of gauge fields on D2-D0 brane bound states 11, 18, 19, 20. On the other hand, D1-brane physics on a two-torus is not Morita equivalent to the D2brane physics. The D1-brane physics is, however, T-dual to the D2-brane (D2-D0) physics. (In the rest of this paper we use the term 'T-duality' in this sense.) Geometrically, the D1-brane picture is simpler, so is used to realize the D2-brane physics and the $S L(2, \mathbb{Z})$ symmetry which acts on the D2-brane physics [21, 22, 23]. The noncommutativity of the algebra of noncommutative two-tori is also understood intuitively in this picture [2]. However, the noncommutativity in the D1-brane picture is not described by the deformation quantization (Moyal-product).

In this paper, the algebra of open string field on the D1-brane is identified with the crossed product representation of noncommutative tori. Namely, a noncommutative torus represented in the deformation quantization picture and the one represented by crossed product are connected by T-duality. On the D1-brane physics, the crossed product is obtained from the geometry of the Kronecker foliation. The Morita equivalence of the algebra of open strings is then realized naturally in this picture. Note that for two-tori the T-dual is equivalent to mirror dual. Homological mirror symmetry [24] is then the open string version of the mirror symmetry [25] from a physical viewpoint. Thus, the results stated above are expected to be relevant to the homological mirror symmetry on two-tori. In fact, from the viewpoints of the results, we succeed to find a natural subcategory of the category of projective modules over noncommutative two-tori which corresponds to a category in homological mirror symmetry setup. The fact might give an insight on the nonperturbative structure over noncommutative tori. The subcategory is closely related to the theory of holomorphic vectors (or theta vectors) introduced by A. Schwarz 26, 27].

This paper is organized as follows. In Section 2, we review the basic facts on the Kronecker foliation. Subsection 2.1 explains the relation between the Kronecker foliation on a two-torus and the crossed product representation of a noncommutative two-torus. The Kronecker foliation provides us an intuitive picture why Morita equivalent noncom- 
mutative two-tori are generated by $S L(2, \mathbb{Z})$, which is explained in Subsection 2.2. In Section 3 we consider D1-branes on the two-torus and the physics of the open string ending on them. Subsection 3.1 shows that the algebra of the open strings on the D1-brane is just the noncommutative two-torus in the crossed product representation explained in Subsection 2.1. The D1-branes can wrap various cycles $S^{1}$ on the two-torus. We discuss the open strings on such D1-branes in Subsection 3.2. Such situations correspond to that in Subsection 2.2 in a certain limit. We discuss in Section 4 when the situations in Subsection 3.2 and Subsection 2.2 coincide. It is shown that both situations coincide in the limit related to the so-called Seiberg-Witten limit [6]. Section 5 presents an application of the results stated above. In Subsection 5.1 projective modules on noncommutative two-tori are realized along the previous arguments. In Subsection 5.2 we relate the theory on the projective modules to the homological mirror symmetry. Namely, noncommutative analogue of the homological mirror symmetry is proposed. Alternatively, the results also mean that some open string interaction in the noncommutative theory can be realized as disk instanton contributions.

\section{Kronecker foliation and Morita equivalence}

Noncommutative tori can be obtained from the Kronecker foliation [16]. We shall review the fact in Subsection 2.1. Such a representation of noncommutative tori admits an intuitive and geometric realization for the Morita equivalence. We shall explain it in Subsection 2.2.

\subsection{Kronecker foliation and noncommutative torus}

Let $\left(x_{1}, x_{2}\right)$ be the coordinates on a two-torus $\mathbb{T}^{2} \simeq \mathbb{R}^{2} / \mathbb{Z}^{2}$ with periodicity $x_{1} \sim x_{1}+1$ and $x_{2} \sim x_{2}+1$. Let us consider the line

$$
x_{2}=\theta x_{1}
$$

on the covering space $\mathbb{R}^{2}$. When the slope $\theta$ is rational, the image of the line on $\mathbb{T}^{2}$ is $S^{1}$. However when $\theta$ is irrational, the image fills densely in $\mathbb{T}^{2}$.

In this paper, we shall discuss the irrational case. The pair of $\mathbb{T}^{2}$ and the line (1) on $\mathbb{T}^{2}$ (Fig.1) is called the Kronecker foliation with the irrational slope. Generally, a manifold $M$ is said to be foliated when we have a partition of $M$ into its submanifolds whose codimension is greater than one. Such submanifolds are then called the leaves of $M$. Here the line Eq.(11) is the leaf of the Kronecker foliation.

Let us parametrize the leaf as $\left(x_{1}, x_{2}\right)=(t, \theta t), t \in \mathbb{R}$. Now we consider $S^{1}$ defined by $x_{1}=0$ and functions on the $S^{1}$. The functions are represented as a Fourier-expanded 
form :

$$
a\left(x_{2}\right)=\sum_{m \in \mathbb{Z}} a_{m} e^{2 \pi i x_{2}}
$$

The generator of these functions is $e^{2 \pi i x_{2}}$ and we define

$$
U_{2}:=e^{2 \pi i x_{2}}
$$

On the covering space of $\mathbb{T}^{2}$, there are the mirror images of $S^{1}$ which are expressed as $x_{1}=c_{1}, c_{1} \in \mathbb{Z}$. Note that the leaf is necessarily transversal to the $S^{1}$ as in Fig.11. The

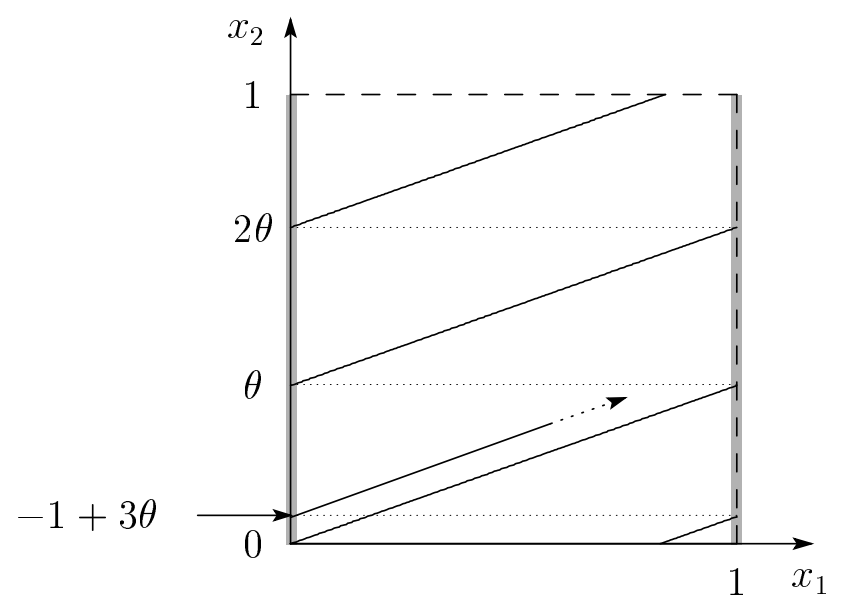

Figure 1: The Kronecker foliation with slope $\theta$. The line of slope $\theta$ is the leaf of the foliation. The leaf is transversal to the cycle $S^{1}$ described by $x_{1}=c_{1}, c_{1} \in \mathbb{Z}$.

point $t=0$ is an intersection point of the leaf and the $S^{1}$, and the leaf then intersects with the $S^{1}$ in the next time at $t=1$, i.e. $\left(x_{1}, x_{2}\right)=(1, \theta)$. Correspondingly, we define the following action $U_{1}$ on the functions on $S^{1}$.

$$
U_{1} a\left(x_{2}\right)=a\left(x_{2}+\theta\right)
$$

These two generators satisfy the following relation

$$
U_{1} U_{2}=e^{2 \pi i \theta} U_{2} U_{1}
$$

Thus, the algebra generated by $U_{1}$ and $U_{2}$ is the noncommutative two-torus. Such a representation of the noncommutative torus is called the crossed product representation of the rotation algebras 16. 


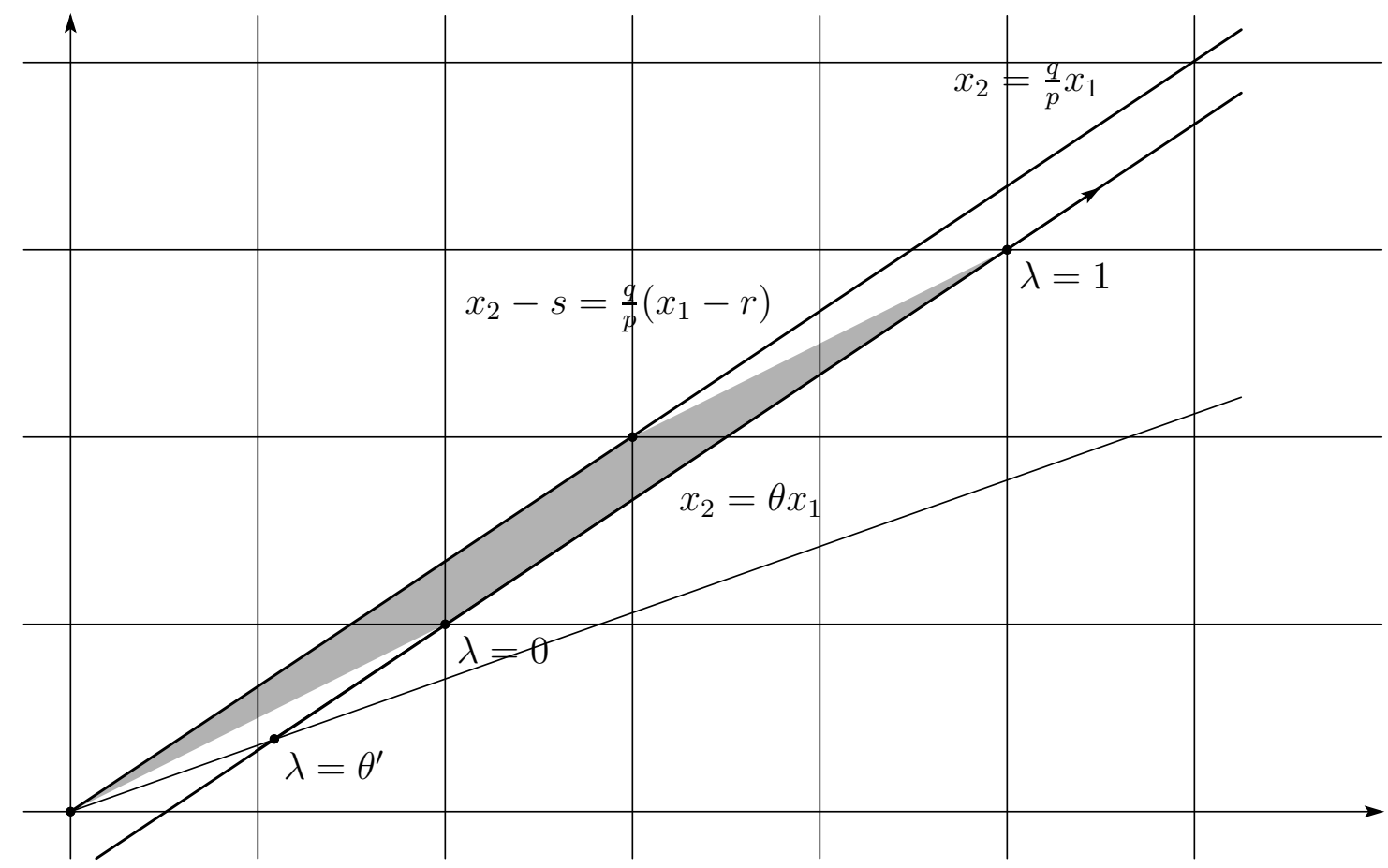

Figure 2: The unit square denotes the unit area of the two-torus. On its covering space, the line $x_{2}=\frac{q}{p} x_{1}$ expresses a cycle $S^{1}$ which winds $p$ times in $x_{1}$ direction and $q$ times in $x_{2}$ direction on the two-torus. This is the figure in the case of $(p, q)=(3,2)$ and $(r, s)=(2,1)$. Since $q r-p s=1$, the parallelogram spanned by $(p, q)$ and $(r, s)$ describes a unit area of another new two-torus.

\subsection{Kronecker foliation and Morita equivalence}

One can take other cycle $S^{1}$ which is transversal to the leaf of the foliation. They are characterized by relatively prime integers $p$ and $q$. The cycle is expressed by the line $x_{2}=q x_{1} / p$ on the covering space $\mathbb{R}^{2}$ of the two-torus. The periodicity of the 1 -cycle is $p$ for $x_{1}$ direction and $q$ for $x_{2}$ direction (see Fig.2). Let us introduce $r, s \in \mathbb{Z}$ which satisfy $\left|\begin{array}{ll}r & p \\ s & q\end{array}\right|=1$. The vectors $\left(x_{1}, x_{2}\right)=(r, s)$ and $\left(x_{1}, x_{2}\right)=(p, q)$ on the covering space then define the unit area of the torus. Fig. 2 shows such a situation in the case $(p, q)=(3,2)$ and $(r, s)=(2,1)$. We have an ambiguity of the choice of $(r, s)$ such as

$$
(r, s) \sim(r, s)+\mathbb{Z}(p, q)
$$

However one can see later that this ambiguity has no matter for our problems. Let us consider the action $U_{1}$ on the cycle $S^{1}$ as in the previous subsection. To find the action, it is sufficient to get the point at which the leaf of the foliation $x_{2}=\theta x_{1}$ and the 1-cycle $\left(x_{1}, x_{2}\right)=(r+\lambda p, s+\lambda q), \lambda \in \mathbb{R}$ intersect to each other. The intersecting point is then 
given by

$$
\lambda=\frac{r \theta-s}{q-p \theta} .
$$

Here let $\theta^{\prime}:=\lambda$ and consider the functions on the cycle $S^{1}$ generated by $Z_{2}=e^{2 \pi i z}$. On it $Z_{2}=e^{2 \pi i z}$ acts by multiplication and $Z_{1}: f(z) \mapsto f\left(z+\theta^{\prime}\right)$ acts as translation. $Z_{1}$ and $Z_{2}$ then have the relation

$$
Z_{1} Z_{2}=e^{2 \pi i \theta^{\prime}} Z_{2} Z_{1}, \quad \theta^{\prime}:=\frac{r \theta-s}{q-p \theta} .
$$

We denote by $\mathcal{A}_{\theta^{\prime}}$ the algebra generated by $Z_{1}$ and $Z_{2}$. Note that the ambiguity in Eq.(2) affects $\theta^{\prime}$ as $\theta^{\prime}-\mathbb{Z}$ so the relation (3) is independent of the ambiguity.

It is well-known that the noncommutativity of the Morita equivalent noncommutative tori are related by the fractional transformation as in Eq.(3). Generally, for a given foliation in a manifold $M$, one can define a $C^{*}$-algebra $\mathcal{A}$ associated to it. Furthermore when one considers a closed submanifold $V$ of $M$ which is transversal to the leaf space of the foliation, it is known that $\mathcal{A}$ is Morita equivalent to the algebra $\left.\mathcal{A}\right|_{V}$ which is obtained by 'restricting' $\mathcal{A}$ to $V$. Here $M$ is the two-torus, the foliation is the Kronecker foliation, $V$ is the cycle $S^{1}$ characterized by $(p, q)$, and the algebra $\left.\mathcal{A}\right|_{V}$ is the noncommutative two-torus $\mathcal{A}_{\theta^{\prime}}$ in the crossed product representation. Since the $C^{*}$-algebra $\mathcal{A}$ and noncommutative torus $\mathcal{A}_{\theta^{\prime}}$ is Morita equivalent for any (relatively prime) integers $(p, q)$, we can say that $\mathcal{A}_{\theta^{\prime}}$ is Morita equivalent to $\mathcal{A}_{\theta}$ [16].

If we translate the torus by

$$
\left(\begin{array}{l}
x_{1} \\
x_{2}
\end{array}\right) \rightarrow\left(\begin{array}{l}
x_{1}^{\prime} \\
x_{2}^{\prime}
\end{array}\right)=\left(\begin{array}{ll}
r & p \\
s & q
\end{array}\right)^{-1}\left(\begin{array}{l}
x_{1} \\
x_{2}
\end{array}\right)
$$

so that the vectors $(r, s)$ and $(p, q)$ are transformed to $(1,0)$ and $(0,1)$, respectively, the foliation on the torus is expressed as in Fig.2 but the slope is $\theta^{\prime}$.

In the next section, we relate the cycle $S^{1}$ to a D1-brane, whereas the leaf is identified with the orbit of the open string ending on the D1-brane in a certain limit.

\section{Open string ending on D1-brane}

Let us consider a D1-brane winding on a two-torus. We denote its slope by $q / p$ and for simplicity we assume that it passes the origin. Now we introduce the metric on the twotorus as $\hat{g}=\left(\begin{array}{ll}\hat{g}_{11} & \hat{g}_{12} \\ \hat{g}_{12} & \hat{g}_{22}\end{array}\right)$. The orbit of the open string with both ends on the D1-brane is determined so that the open string minimizes its length with respect to the metric $\hat{g}$. Of course this determination is consistent with the first quantization picture of open strings 23]. 


\section{$3.1 \quad(p, q)=(0,1)$ case}

First, let us find the slope of the open string which minimizes its length in the simplest case $(p, q)=(0,1)$. The situation is just the same as the argument in [2], where the noncommutativity of the product of open string field is realized in a very intuitive and geometric way. The length square of the open string which winds once around $x_{1}$ direction is

$$
\left(\begin{array}{ll}
1 & \lambda
\end{array}\right)\left(\begin{array}{ll}
\hat{g}_{11} & \hat{g}_{12} \\
\hat{g}_{12} & \hat{g}_{22}
\end{array}\right)\left(\begin{array}{l}
1 \\
\lambda
\end{array}\right)
$$

where $\lambda$ is the slope of the orbit of the open string (see Fig. (1). Differentiating this length by $t$, one can easily see that it is minimized by $\lambda=-\hat{g}_{12} / \hat{g}_{22}$. Here set $\theta:=\lambda$ and one can relate this situation to that in Fig.1. The D1-brane is the cycle $S^{1}$. The open string then winds along the leaf of the foliation.

Let us consider field theory on the D1-brane. Note that the noncommutative product is not described by the Moyal product (*-product). We shall below explain that the noncommutative product structure can be identified with the crossed product defined in the previous section. The field corresponding to the open string ending on the D1-brane has two kinds of modes, the momentum along the D1-brane and the winding modes. Both modes are discretized and take their values in $\mathbb{Z}$. Let $m$ and $w$ be the momentum and the winding, respectively. The field $\phi$ can be Fourier-expanded by $m$ as

$$
\phi\left(x_{2}\right)=\sum_{m \in \mathbb{Z}} \phi_{m} U_{2}^{m}
$$

where $U_{2}=e^{2 \pi i x_{2}}$. Furthermore $\phi_{m}$ is expanded by $w$ as $\phi_{m}=\sum_{w \in \mathbb{Z}} \phi_{w, m} U_{1}^{w}$ where $U_{1}$ is the generator defined in the previous section.

Actually this expression of the field agrees with the open string physics as explained below. The product of the fields is identified with the open string interaction. When two open strings with modes $(w, m)$ and $\left(w^{\prime}, m^{\prime}\right)$ interact, an open string is created and its modes should simply be the sum of the two open string modes. Correspondingly, now the product of $U_{1}^{w} U_{2}^{m}$ with $U_{1}^{w^{\prime}} U_{2}^{m^{\prime}}$ in fact coincides with $U_{1}^{w+w^{\prime}} U_{2}^{m+m^{\prime}}$ up to a coefficient (phase factor). Moreover, the interaction of two open strings is defined by joining the end points of them. The open string with winding $w$ stretches with length $\theta w$ in $x_{2}$ direction. Therefore if the open string with modes $(w, m)=(1,0)$ acts on the function on the D1brane $f\left(x_{2}\right)$, the next open string acts on $f\left(x_{2}+\theta\right)$. This justifies the identification of the generator of the winding modes with $U_{1}$ defined in the previous section. The open string vertex is then described as the closed loop of the open strings. It in fact closes due to the momentum and winding modes conservation. The 'integral' of the fields are then defined 
by

$$
\operatorname{Tr}\left(\sum_{w, m \in \mathbb{Z}^{2}} \phi_{w, m} U_{1}^{w} U_{2}^{m}\right)=\phi_{0,0}
$$

This is the standard definition of the 'integral' on noncommutative two-tori.

Thus, the field theory can be expressed as noncommutative two-tori.

\subsection{General $(p, q)$ case}

Next we shall consider the D1-brane winding on general 1-cycles on the two-torus and discuss the relation to the Morita equivalence. We express the D1-brane as $x_{2}=q x_{1} / p$ where $p$ and $q$ are relatively prime integers (see Fig.2). As in the previous subsection, we shall find the orbit of the open string ending on the D1-brane. We take $r, s \in \mathbb{Z}$ which satisfy $\left|\begin{array}{ll}r & p \\ s & q\end{array}\right|=1$, and consider the open string ending on $\left(x_{1}, x_{2}\right)=(0,0)$ and $\left(x_{1}, x_{2}\right)=(r+\lambda p, s+\lambda q)$. The latter is expressed as

$$
\left(\begin{array}{l}
x_{1} \\
x_{2}
\end{array}\right)=\left(\begin{array}{l}
r+\lambda p \\
s+\lambda q
\end{array}\right)=\left(\begin{array}{ll}
r & p \\
s & q
\end{array}\right)\left(\begin{array}{l}
1 \\
\lambda
\end{array}\right) .
$$

Its length square with respect to the metric $\hat{g}$ is then given by

$$
\left(\begin{array}{ll}
r+\lambda p & s+\lambda q
\end{array}\right) \hat{g}\left(\begin{array}{l}
r+\lambda p \\
s+\lambda q
\end{array}\right)=\left(\begin{array}{ll}
1 & \lambda
\end{array}\right) \hat{g}^{\prime}\left(\begin{array}{l}
1 \\
\lambda
\end{array}\right)
$$

where

$$
\hat{g}^{\prime}=\left(\begin{array}{ll}
\hat{g}_{11}^{\prime} & \hat{g}_{12}^{\prime} \\
\hat{g}_{12}^{\prime} & \hat{g}_{22}^{\prime}
\end{array}\right)=\left(\begin{array}{ll}
r & p \\
s & q
\end{array}\right)^{t}\left(\begin{array}{ll}
\hat{g}_{11} & \hat{g}_{12} \\
\hat{g}_{12} & \hat{g}_{22}
\end{array}\right)\left(\begin{array}{ll}
r & p \\
s & q
\end{array}\right) .
$$

Thus the argument reduces to the $(p, q)=(0,1)$ case. The length of the open string is minimized at

$$
\lambda=-\frac{\hat{g}_{12}^{\prime}}{\hat{g}_{22}^{\prime}}
$$

and the slope of the orbit of the open string is then

$$
\frac{s+\lambda q}{r+\lambda p}=-\frac{p \hat{g}_{11}+q \hat{g}_{12}}{p \hat{g}_{12}+q \hat{g}_{22}} .
$$

We would like to relate this slope (5) to that of the leaf of the foliation in Subsection 2.2. However, the slope (5) depends on $p$ and $q$ in general $\hat{g}$. Now let us consider the degenerate metric on the two-torus of the form

$$
\hat{g}=C\left(\begin{array}{cc}
\theta^{2} & -\theta \\
-\theta & 1
\end{array}\right)
$$


where $C$ is a constant. Since the metric is degenerate, the length of the open string along the vector $(a, b) \in \mathbb{R}^{2}$ is the square root of

$$
\left(\begin{array}{ll}
a & b
\end{array}\right) \hat{g}\left(\begin{array}{l}
a \\
b
\end{array}\right)=C(a \theta-b)^{2} .
$$

Clearly the mass of any open string stretching with slope $\theta$ is zero. In this case, the slope (5) is independent of $(p, q)$ and coincides with $\theta$. Therefore the situation just coincides with that in Subsection 2.2. The open string field is represented as $\phi=\sum_{(w, m) \in \mathbb{Z}^{2}} \phi_{w, m} Z_{1}^{w} Z_{2}^{m}$. Here $Z_{1}$ and $Z_{2}$ are two generators of the noncommutative two-torus. They have the relation $Z_{1} Z_{2}=e^{2 \pi i \theta^{\prime}} Z_{2} Z_{1}$ where $\theta^{\prime}$ is the one defined in Eq.(3).

Thus we can conclude as follows. The two-torus with metric of the form in Eq.(6) is implicitly 'foliated'. When we probe the physics on the two-torus by a D1-brane on a 1-cycle, the two-torus is 'foliated' by the open strings ending on the D1-brane. Field theory of open strings on the two-torus is described by the noncommutative two-torus in the crossed product representation. Different choices of the 1-cycle on which the probe D1-brane lies are related by the Morita equivalence of the noncommutative two-tori.

The above fact implies that the modular transformation $S L(2, \mathbb{Z})$ preserves the form of the metric (6). Actually,

$$
\left(\begin{array}{ll}
r & p \\
s & q
\end{array}\right)^{t}\left(\begin{array}{cc}
\theta^{2} & -\theta \\
-\theta & 1
\end{array}\right)\left(\begin{array}{ll}
r & p \\
s & q
\end{array}\right)=(q-p \theta)^{2}\left(\begin{array}{cc}
\theta^{\prime 2} & -\theta^{\prime} \\
-\theta^{\prime} & 1
\end{array}\right)
$$

where $\theta^{\prime}=(r \theta-s)(q-p \theta)^{-1}$.

\section{The Seiberg-Witten limit and the Kronecker folia- tion limit}

In this section, we discuss the meaning of the metric (6). Such forms of the metric are related to the so-called Seiberg-Witten limit[6]. The theory of D1-brane on the two-torus is obtained by T-dualizing the theory of D2-brane on the dual torus. Let $E:=g+B$ be

the background (the pair of the metric $g$ and the $B$-field) on the torus where a D2-brane exists. The background $\hat{E}$ of the torus which is obtained by T-dualizing the D2-brane theory is then

$$
\hat{E}=\left(I_{2} E+I_{1}\right)\left(I_{1} E+I_{2}\right)^{-1}, \quad I_{1}:=\left(\begin{array}{ll}
1 & 0 \\
0 & 0
\end{array}\right), \quad I_{2}:=\left(\begin{array}{ll}
0 & 0 \\
0 & 1
\end{array}\right) .
$$

More explicitly, the background $\hat{E}:=\hat{g}+\hat{B}$ is given by

$$
\hat{g}+\hat{B}=g_{11}^{-1}\left(\begin{array}{cc}
1 & B \\
B & \operatorname{det}(g)+B^{2}
\end{array}\right)+g_{11}^{-1}\left(\begin{array}{cc}
0 & -g_{12} \\
g_{12} & 0
\end{array}\right) .
$$


In the Seiberg-Witten limit $g \rightarrow 0$, the above metric reduce to

$$
\hat{g} \sim g_{11}^{-1} B^{2}\left(\begin{array}{cc}
\frac{1}{B^{2}} & \frac{1}{B} \\
\frac{1}{B} & 1
\end{array}\right) .
$$

This is nothing but the metric in Eq.(6) with $C=g_{11}^{-1} B^{2}$ and $\theta=-\frac{1}{B}$. It is known that in the Seiberg-Witten limit the correlation function of open strings with both ends on D2-brane on a two-torus reduces to the algebra of the noncommutative torus [6]. The noncommutativity of the geometry in the Seiberg-Witten limit can be realized from the viewpoints of the deformation quantization. Alternatively, by T-dualizing one direction from a two-torus in the Seiberg-Witten limit, one obtains a two-torus which is degenerate for one direction. Such a geometry is directly related to the Kronecker foliation. D-branes are then transformed to D1-branes winding various direction. The physics of the open strings on the D1-brane can also be represented by the noncommutative torus, but in this side the noncommutativity is expressed in the crossed product. Thus, we can conclude that the two representations of the noncommutative two-tori, that in the deformation quantization and that in the crossed product, are related by T-dual.

\section{Application}

On noncommutative tori, there exist explicit projective modules called Heisenberg modules 28. Projective modules are noncommutative analogue of vector bundles. Therefore projective modules on two-tori correspond to noncommutative D2-branes (more precisely D2-D0 bound states). However the Heisenberg modules are constructed in the crossed product representation. Therefore they also have a D1-brane picture. In Subsection 5.1 we shall show that the Heisenberg modules can be defined from the viewpoint of D1-brane physics. Then in Subsection 5.2 we shall discuss the relation of these arguments to the homological mirror symmetry.

\subsection{Projective modules from the Kronecker foliation}

\section{- Heisenberg modules from D1-brane picture}

In Subsection 2.1, for a noncommutative two-torus $\mathcal{A}_{\theta}$, a Morita equivalent noncommutative two-torus $\mathcal{A}_{\theta^{\prime}}$ was constructed geometrically. Two algebras $\mathcal{A}$ and $\mathcal{A}^{\prime}$ are said to be Morita equivalent when there exists a projective module $E$ such that $\mathcal{A}^{\prime} \simeq$ End $d_{\mathcal{A}} E$. Here $E n d_{\mathcal{A}} E$ denotes the endomorphism algebra of $E$ which commutes with $\mathcal{A}$. Such a projective module $E$ is then called a Morita equivalent bimodule. For noncommutative two-tori, Heisenberg modules are the Morita equivalent bimodule. It is used to prove that $\mathcal{A}_{\theta}$ and $\mathcal{A}_{\theta^{\prime}}$ defined in Eq.(3) are Morita equivalent(see[9, 10, 28]). Such a Heisenberg module over $\mathcal{A}_{\theta}$ is characterized by $\mathcal{A}_{\theta^{\prime}}$ or equivalently $\left(\begin{array}{cc}r & p \\ s & q\end{array}\right) \in S L(2, \mathbb{Z})$, so we denote it 
by $E_{q, p, \theta}$. It is known that any finitely generated projective module is isomorphic to the direct sum of these Heisenberg modules (see [17, 28]).

We shall construct the Heisenberg modules from the D1-brane picture below. As seen in Section 2, the algebra $\mathcal{A}_{\theta}$ is defined on the D1-brane of $(p, q)=(0,1)$ on the two-torus foliated with slope $\theta$. Thus we first fix the $S^{1}$ characterized by $(p, q)=(0,1)$ as the 'base space'. Let us construct module $E_{q, p, \theta}$ with relatively prime $p, q$. In this case, the

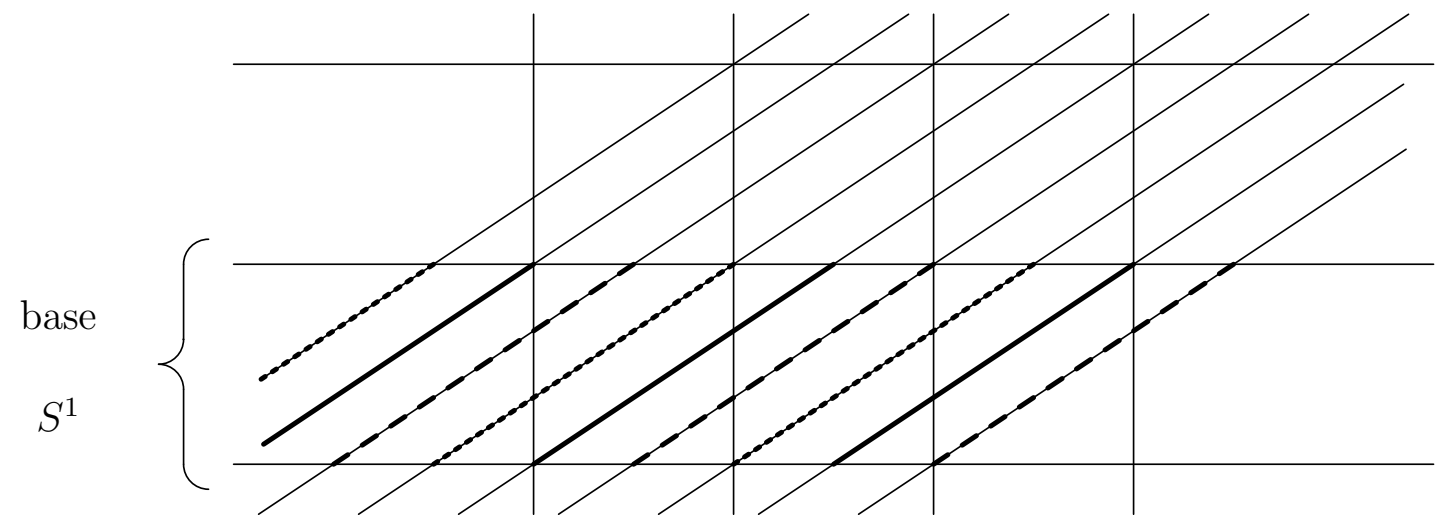

Figure 3: A graphical viewpoint of the Heisenberg module in the case $(p, q)=(3,2)$. There exist $p$ spirals over the base $S^{1}$.

endomorphism of the module $E_{q, p, \theta}$ is $\mathcal{A}_{\theta^{\prime}}$, that is, $\operatorname{End}_{\mathcal{A}_{\theta}} E_{q, p, \theta}$ is essentially that obtained in Subsection 3.2 (Eq.(17)). Therefore we first consider the cycle characterized by $(p, q)$ on the covering space of the two-torus and regard it as lines over the base $S^{1}$. We have lines $x_{1}=p x_{2} / q+l / q$ where $l \in \mathbb{Z}$ but, on the $S^{1}$, the line of $x_{1}$-axis $l / q$ coincides with that of $x_{1}$-axis $(l+p) / q$. Thus we have $p$ number of spirals $\mathbb{R}$ over the $S^{1}$ as in Fig. 3 and then consider a module of $p$ elements. We express it as

$$
f(z, \mu), \quad z \in \mathbb{R} \quad \mu=0,1, \cdots, p-1 .
$$

Here we identify the value $f(0, \mu)$ with the value of a function at $\left(x_{1}, x_{2}\right)=\left(0, \frac{\mu q}{p}\right)$ on $(p, q)$ D1-brane. When we define the operation of $U_{i}$ 's on this module, $z$ is regarded as the coordinate on the base $S^{1}$ with period $z \sim z+1$. Namely, the value of the function at point $\left(x_{1}, x_{2}\right)=\left(\frac{p z}{q}, z+\frac{\mu q}{p}\right)$ is regarded as $f(z, \mu)$. $U_{2}$ is essentially the generator of functions on the $S^{1}$ as $U_{2}=e^{2 \pi i z}$ in Subsection 2.1. However, now the action of $U_{2}$ should preserve the relation $f\left(z=\frac{q}{p}, 0\right)=f(0, \mu=1)$ because $f\left(\frac{q}{p}, 0\right)$ and $f(0,1)$ are the values of the function at the same points on the D1-brane. In this way the action of $U_{2}$ is determined as

$$
U_{2} f(z, \mu)=f(z, \mu) e^{2 \pi i\left(z+\mu \frac{q}{p}\right)} .
$$

\footnotetext{
${ }^{2}$ One can see later that actually the Heisenberg modules do not depend on the ambiguity of $(r, s)$ in $\mathrm{Eq} \cdot(2)$.
} 
The action of $U_{1}$ is also given in the same way as in Subsection 2.1, but we define it so that the open string shifts $\mu$ to $\mu+1$. The open string starting at $\left(x_{1}, x_{2}\right)=(0, z)$ then winds around $x_{2}$ direction and ends at $\left(x_{1}, x_{2}\right)=(0, z+\theta)$ on the base $S^{1}$, and $z+\theta$ is $z+\theta-\frac{q}{p}$ in the shifted coordinate. Thus $U_{1}$ is

$$
U_{1} f(z, \mu)=f\left(z-\frac{q}{p}+\theta, \mu+1\right) .
$$

On the other hand, $\mathcal{A}_{\theta^{\prime}}$ acts on the lines with slope $q / p$. The action of $Z_{i}$ is essentially that discussed in Subsection 2.2. However, due to the definition of the endomorphism, the action of $\mathcal{A}_{\theta^{\prime}}$ must commute with $U_{1}$ and $U_{2}$. It can be accomplished by identifying the coordinate on the lines of slope $q / p$ with the coordinate $z$ on the base $S^{1}\left(x_{1}=0\right)$ as follows. For each $\mu$, we consider the open string stretching between the line $x_{1}=0$ and the line $x_{2}=\frac{q x_{1}}{p}+\left(\frac{q \mu}{p}+\mathbb{Z}\right)$ on the covering space. The open string starting at $\left(x_{1}, x_{2}\right)=\left(0, z+\frac{q \mu}{p}+\mathbb{Z}\right)$ ends at point $\left(x_{1}, x_{2}\right)=\left(\frac{p z}{q-p \theta}, \frac{q z}{q-p \theta}+\frac{q \mu}{p}+\mathbb{Z}\right)$. The corresponding coordinate on the line $x_{2}=\frac{q x_{1}}{p}+\left(\frac{q \mu}{p}+\mathbb{Z}\right)$ is then defined by $z$.

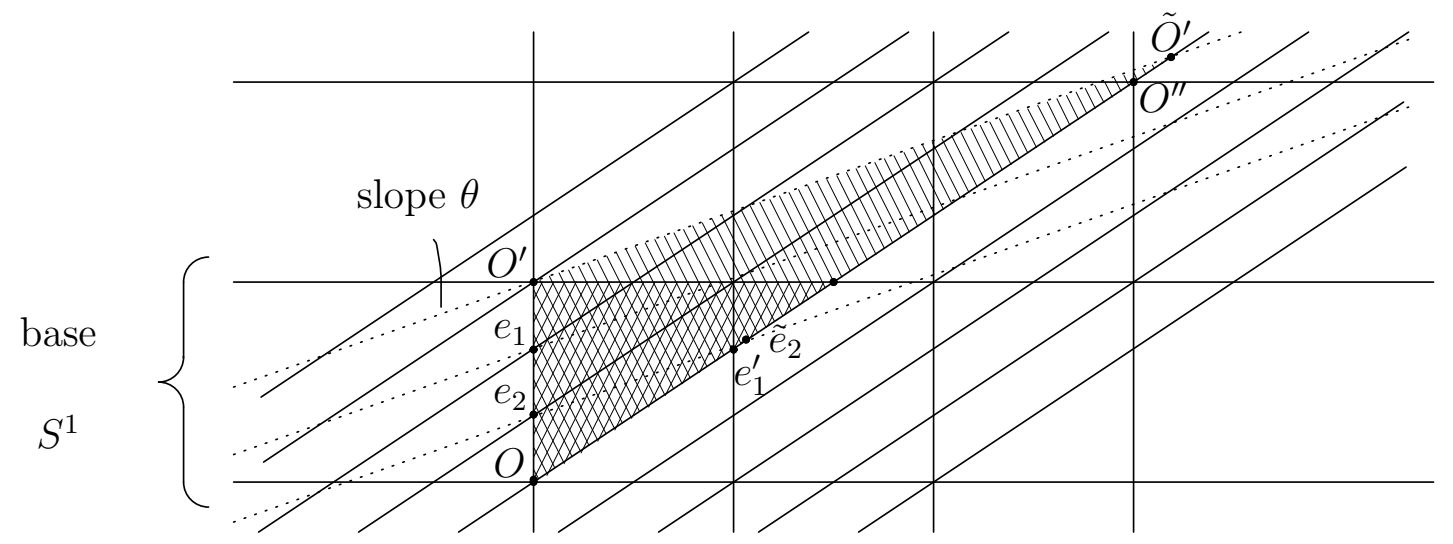

Figure 4: $U_{i}$ acts on cycle $x_{1}=0$ and $Z_{i}$ acts on the cycle characterized by $(p, q)$. For the action of $U_{i}, z$ in the expression of the module $f(z, \mu)$ can be regarded as the coordinate on $x_{1}=0$ with its periodicity $z \sim z+1$. In this figure, $\left|O O^{\prime}\right|=1$. While, $z$ is regarded as the coordinate on $x_{2}=\frac{q x_{1}}{p}+\left(\frac{q \mu}{p}+\mathbb{Z}\right)$ when $Z_{i}$ acts on $f(z, \mu)$. Its periodicity is defined so that the action of $\mathcal{A}_{\theta}$ commutes with that of $\mathcal{A}_{\theta^{\prime}}$. Then $\left|O \tilde{O}^{\prime}\right|=1$ and the period is $\left|O O^{\prime \prime}\right|=q-p \theta$.

With this coordinate, the period of the D1-brane is $q-p \theta$ (see Fig.(A). One can also see that $\left(z=(q-p \theta) \frac{1}{p}, \mu\right)$ and $(z=0, \mu+1)$ represent the same point on the D1-brane. For instance for $\mu=0, e_{1}^{\prime}$ and $e_{1}$ in Fig. $₫$ is the same point. Thus, we define

$$
Z_{2} f(z, \mu)=f(z, \mu) e^{2 \pi i\left(\frac{z}{q-p \theta}+\frac{\mu}{p}\right)} .
$$

Moreover, an open string ending at a point $z$ on the D1-brane $\mu$ starts from the point $z-\frac{1}{p}$ on the D1-brane $\mu+r$ up to $p \mathbb{Z}$. In Fig. 1 for $\mu=0$ the open string ending at $\tilde{e}_{2}$ 
starts from $e_{2}$. Therefore we define

$$
Z_{1} f(z, \mu)=f\left(z-\frac{1}{p}, \mu+r\right) .
$$

The action of $U_{i}$ and $Z_{i}$ for the same $i=1,2$ commutes trivially, and the action of $U_{i}$ and $Z_{i}$ for different $i$ commutes by the definition of the coordinate.

To summarize, we have the following representation of the generators of algebra $\mathcal{A}_{\theta}$ and $\mathcal{A}_{\theta^{\prime}}$.

$$
\begin{aligned}
& U_{1} f(z, \mu)=f\left(z-\frac{q}{p}+\theta, \mu+1\right) \\
& U_{2} f(z, \mu)=f(z, \mu) e^{2 \pi i\left(z+\mu \frac{q}{p}\right)} \\
& Z_{1} f(z, \mu)=f\left(z-\frac{1}{p}, \mu+r\right) \\
& Z_{2} f(z, \mu)=f(z, \mu) e^{2 \pi i\left(\frac{z}{q-p \theta}+\frac{\mu}{p}\right)}
\end{aligned}
$$

In this expression, $U_{i}$ and $Z_{i}$ satisfy the following relation

$$
U_{1} U_{2}=e^{2 \pi i \theta} U_{2} U_{1}, \quad Z_{1} Z_{2}=e^{2 \pi i\left(-\theta^{\prime}\right)} Z_{2} Z_{1} .
$$

We define on this Heisenberg module the action of $U_{i}$ as left action and that of $Z_{i}$ as right action. Therefore, the sign of $-\theta^{\prime}$ flips. That is, this module is regarded as a $\mathcal{A}_{\theta^{-}} \mathcal{A}_{\theta^{\prime}}$ bimodule. As mentioned in Eq.(2), the choice of $(r, s)$ has an ambiguity when relatively prime integers $p, q$ are given. However one can confirm from Eq.(11) that the module $E_{q, p, \theta}$ does not depend on the ambiguity since $\mu$ is defined up to $p \mathbb{Z}$.

It is known that the module obtained above equips the following constant curvature connection.

$$
\nabla_{1}=\frac{2 \pi i p}{q-p \theta} z, \quad \nabla_{2}=\frac{\partial}{\partial z}
$$

Thus, modules $E_{q, p, \theta}$ has a constant curvature $\left[\nabla_{1}, \nabla_{2}\right]=\frac{2 \pi i p}{q-p \theta}$.

The Heisenberg modules constructed above are regarded as that defined on the base space $S^{1}$ characterized by $(0,1)$. These can be generalized easily in the case that $(0,1)$ is replaced to any (relatively prime) $(p, q)$. In this case the modules are characterized by two cycles $x_{2}=\frac{q}{p} x_{1}$ and $x_{2}=\frac{Q}{P} x_{1}$. The corresponding module can be constructed in a quite similar way as the $(p, q)=(0,1)$ case above. Consequently one obtains Heisenberg module $E_{q^{\prime}, p^{\prime}, \theta^{\prime}}$ over $\mathcal{A}_{\theta^{\prime}}$ where

$$
\left(\begin{array}{ll}
R & P \\
S & Q
\end{array}\right)=\left(\begin{array}{ll}
r & p \\
s & q
\end{array}\right)\left(\begin{array}{ll}
r^{\prime} & p^{\prime} \\
s^{\prime} & q^{\prime}
\end{array}\right),
$$

\footnotetext{
${ }^{3}$ This construction of Heisenberg modules is standard (see [28]). However, the sign convention for $\mu$ is reversed compared to [28] so that the convention for Heisenberg modules agree with the one for an $A_{\infty}$-category in 29]. See the next subsection.
} 
or equivalently $\mathcal{A}_{\theta^{\prime}}-\mathcal{A}_{\theta^{\prime \prime}}$ bimodules where $\theta^{\prime \prime}:=\frac{R \theta-S}{Q-P \theta}$ (see Fig.5).

- The tensor product

The module $E_{q, p, \theta}$ constructed above can also be regarded as a $\mathcal{A}_{\theta^{-}} \mathcal{A}_{\theta^{\prime}}$ bimodule. More generally, as mentioned above, a module characterized by the pair $x_{1}=p x_{2} / q$ and $x_{1}=P x_{2} / Q$ belongs to a $\mathcal{A}_{\theta^{\prime}}-\mathcal{A}_{\theta^{\prime \prime}}$ bimodule. Note that bimodules are regarded as open strings [6, 13]. The line $x_{1}=p x_{2} / q\left(\right.$ resp. $\left.x_{1}=P x_{2} / Q\right)$ characterizes module $E_{p, q, \theta}$ (resp. $\left.E_{P, Q, \theta}\right)$ over $\mathcal{A}_{\theta}$ by fixing the base $S^{1}$ as $x_{1}=0$. The module $E_{p, q, \theta}$ corresponds to the bound state of $p$ D2-branes and $-q$ D0-branes and the module $E_{P-Q \theta}$ also does similarly. The $\mathcal{A}_{\theta^{\prime}}-\mathcal{A}_{\theta^{\prime \prime}}$ bimodule is then regarded as the open string stretching between the D-branes $E_{p, q, \theta}$ and $E_{P, Q, \theta}$.

Now, let us consider the D-branes represented by the lines $x_{1}=0, x_{1}=p x_{2} / q$ and $x_{1}=P x_{2} / Q$. We have the open strings which belong to a $\mathcal{A}_{\theta^{-}} \mathcal{A}_{\theta^{\prime}}$ bimodule $E_{q, p, \theta}$ and a $\mathcal{A}_{\theta^{\prime}}-\mathcal{A}_{\theta^{\prime \prime}}$ bimodule $E_{q^{\prime}, p^{\prime}, \theta^{\prime}}$. These open strings interact on the D1-brane $x_{1}=p x_{2} / q$ and then become an open string stretching between the D1-brane $x_{1}=0$ and $x_{1}=P x_{2} / Q$. Then the open string should be expressed as an element of a $\mathcal{A}_{\theta^{-}} \mathcal{A}_{\theta^{\prime \prime}}$ bimodule $E_{Q, P, \theta}$.

Such open string interactions can be accomplished by the tensor product between bimodules. The tensor product between $E_{q, p, \theta}$ and $E_{q^{\prime}, p^{\prime}, \theta^{\prime}}$ is defined so that $E_{q, p, \theta} a \otimes_{\mathcal{A}_{\theta^{\prime}}}$ $E_{q^{\prime}, p^{\prime}, \theta^{\prime}} \sim E_{q, p, \theta} \otimes_{\mathcal{A}_{\theta^{\prime}}} a E_{q^{\prime}, p^{\prime}, \theta^{\prime}}$ for $a \in \mathcal{A}_{\theta^{\prime}}$. Fig. 5 shows a geometric picture of the tensor product in the case $\left(\begin{array}{ll}r & p \\ s & q\end{array}\right)=\left(\begin{array}{ll}1 & 1 \\ 0 & 1\end{array}\right)$ and $\left(\begin{array}{ll}r^{\prime} & p^{\prime} \\ s^{\prime} & q^{\prime}\end{array}\right)=\left(\begin{array}{ll}1 & 2 \\ 0 & 1\end{array}\right)$. Let $\varphi$ be the isomorphism from $E_{q, p, \theta} \otimes_{\mathcal{A}_{\theta^{\prime}}} E_{q^{\prime}, p^{\prime}, \theta^{\prime}}$ to $E_{Q, P, \theta}$. Such an isomorphism is recently constructed explicitly in [27] f. It is given by

$$
\varphi(f \otimes g)(z, \rho)=\sum_{u \in \mathbb{Z}} f\left(z+\frac{1}{p}\left(u-\frac{p^{\prime}}{P} \rho\right),-r u+\rho\right) \cdot g\left(\frac{z}{q-p \theta}-\frac{q^{\prime}-p^{\prime} \theta^{\prime}}{p^{\prime}}\left(u-\frac{p^{\prime}}{P} \rho\right), u\right) .
$$

Actually, one can check that this tensor product satisfies

$$
\varphi\left(\left(Z_{i} f\right) \otimes g\right)=\varphi\left(f \otimes\left(U_{i} g\right)\right)
$$

for $i=1,2$. Moreover it is defined so that

$$
\begin{aligned}
& \varphi\left(\left(U_{i} f\right) \otimes g\right)=U_{i} \varphi(f \otimes g) \\
& \varphi\left(f \otimes\left(Z_{i} g\right)\right)=Z_{i} \varphi(f \otimes g)
\end{aligned}
$$

for $i=1,2$. In the above equations, $U_{i}$ 's in the left hand side are those which act on $E_{q, p, \theta}$ and $U_{i}$ 's in the right hand side are those which act on $E_{Q, P, \theta}$. Similarly $Z_{i}$ 's in both sides are different from each other.

\footnotetext{
${ }^{4}$ Precisely, the tensor product constructed in [27] is different from that presented here. In [27] the tensor product between right $\mathcal{A}_{\theta}$ modules and left $\mathcal{A}_{\theta}$ modules is constructed. In that case, for right $\mathcal{A}_{\theta}$ module $E_{q, p, \theta}$ and left $\mathcal{A}_{\theta}$ modules $E_{q^{\prime}, p^{\prime}, \theta}$, the tensor product is defined so that $E_{q, p, \theta} U_{i} \otimes_{\mathcal{A}_{\theta}} E_{q^{\prime}, p^{\prime}, \theta} \sim$ $E_{q, p, \theta} \otimes_{\mathcal{A}_{\theta}} U_{i} E_{q^{\prime}, p^{\prime}, \theta}$ where $U_{i}$ 's are two generators of $\mathcal{A}_{\theta}$. We instead define the tensor product as in Eq.(15) for our purposes.
} 


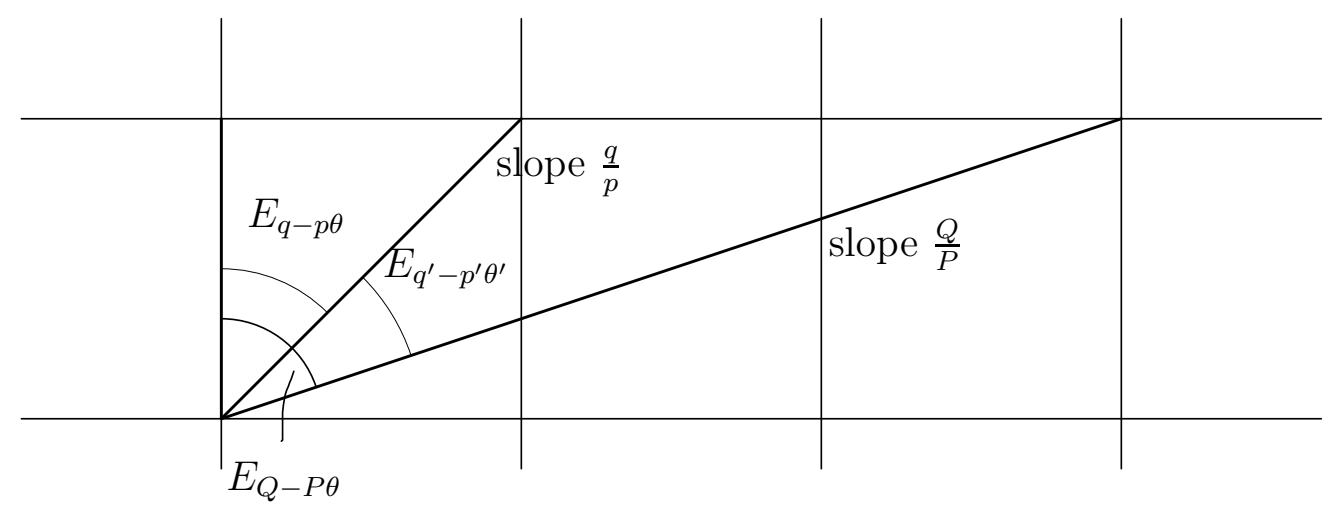

Figure 5: The graphical picture of the tensor product in the case of $(p, q)=(1,1)$ and $\left(p^{\prime}, q^{\prime}\right)=(1,2)((P, Q)=(1,3))$. Each module is characterized by the pair of rational slopes of lines.

\subsection{Relation to the homological mirror symmetry}

It is known that the two-torus with background $E=g+B$ is mirror dual to the T-dual torus which has background $\hat{E}=\hat{g}+\hat{B}$ (Eq.(8)). Mirror symmetry is a symmetry between Calabi-Yau manifolds. It is related to a closed string physics as well as the T-duality is. Instead, one can also consider D-branes on the manifolds and the (topological) open strings between them[25]. These tools may enable us to realize the symmetry between mirror manifolds. Homological mirror symmetry proposal 24 is a mathematical setup of such an attempt. On Calabi-Yau manifolds one can define two categories which are related to the topological open string (A-model or B-model) in [25]. Then the conjecture is that a category on a Calabi-Yau manifold $M$ is equivalent to another category on the mirror dual Calabi-Yau manifold $\hat{M}$. Note that a category consists of objects and morphisms between the objects, where the composition of morphisms satisfies the associativity. One can identify the objects as some kind of D-branes and the morphisms as open strings between them. The category related to the A-model is an $A_{\infty}$-category 0 . Essentially it is constructed by (pseudo) holomorphic map of open string disks. On the other hand, the category related to the B-model is the derived category 10 of coherent sheaves. This side is better understood physically [32, 33, 34]. It is then conjectured that the bounded derived category of coherent sheaves on $M$ is equivalent to the bounded derived category

\footnotetext{
${ }^{5} \mathrm{An} A_{\infty}$-category is a generalization of the usual category. It consists of objects and morphisms, but generally the composition of the morphisms is not associative. Instead, a $A_{\infty}$-category has $i$-linear maps $m_{i}$ of the morphisms for $i \geq 1$ and they satisfy certain relations. The relations contain a condition for $m_{2}$, and it reduces to the associativity relation of $m_{2}$ when $m_{1}=0$. The $A_{\infty}$-category which we shall consider is just in this case, so it is also the usual category with $m_{2}$ the composition of the morphisms.

${ }^{6}$ For the definition of the derived category, see [30]. A brief introduction of it for physicists, see the review part of 31 for example. This paper does not need the precise definition.
} 
of a suitable $A_{\infty}$-category \ and vice visa. In two-tori case, this statement is proved in [29]. We shall avoid explaining the precise statements, and review shortly a part of the story which is relevant here. For the two-torus with background $E=g+B$, the complex structure $\tau$ and the complexified symplectic structure $\rho$ are defined as [36]

$$
\tau=\frac{g_{12}}{g_{11}}+i \frac{\sqrt{g}}{g_{11}}, \quad \rho=i \sqrt{g}+B
$$

Thus, on two-tori, the pairs of the complex structure and the complexified symplectic structure are in one-to-one correspondence with the backgrounds $E=g+B$. The mirror dual torus is defined so that the complex structure and the complexified symplectic structure are interchanged, that is, $\hat{\tau}=\rho$ and $\hat{\rho}=\tau$ where $\hat{\tau}$ and $\hat{\rho}$ are the complex structure and the complexified symplectic structure, respectively, on the mirror dual torus. One can see that the complex and the complexified symplectic structure on the mirror dual torus ( $\hat{\tau}$ and $\hat{\rho}$, respectively) are nothing but those defined by the T-dual background $\hat{E}=\hat{g}+\hat{B}$ in Eq. (8).

Let us consider the two categories; the derived category of coherent sheaves is defined by using the complex structure and is independent of the complexified symplectic structure, while the $A_{\infty}$-category depends only on the complexified symplectic structure. Then the arguments of [29] are as follows. For the bounded derived category of coherent sheaves on the two-torus with complex structure $\tau$, holomorphic vector bundles (and skyscraper sheaf) are considered as the objects and homomorphisms between them are then constructed as the morphisms. It is known that holomorphic sections of vector bundles on tori are described by theta functions. These holomorphic sections are homomorphisms between trivial bundles and the corresponding vector bundles, and in fact any other homomorphism is also described by theta functions. Between these homomorphisms expressed by theta functions, there is a product structure which is given simply by multiplication of two functions on the covering space of the two-torus. On the other hand, on the dual torus with complexified symplectic structure $\hat{\rho}(=\tau)$, an $A_{\infty}$-category is defined as follows. An object of the $A_{\infty}$-category is a pair of a special lagrangian submanifold and a flat bundle on it. Here special lagrangian submanifolds on the two-torus are given by geodesic cycles $S^{1}$, i.e. the geodesic lines on the covering space. If the flat bundle is a line bundle, the object is just the D1-brane discussed until now. An object with rank $n$ flat bundle is then $n$ D1-branes on a cycle $S^{1}$. The morphisms of the $A_{\infty}$-category are then the homomorphism between two flat bundles on each geodesic cycle $S^{1}$. Thus, they

\footnotetext{
${ }^{7}$ In fact, it is still unknown precisely that what kind of $A_{\infty}$-category should correspond to the bounded derived category of coherent sheaves for general (Calabi-Yau) manifolds. An $A_{\infty}$-category is constructed in [35] and refined in 24 to propose the homological mirror symmetry. However generally, the bounded derived category of coherent sheaves is 'larger'. It is then believed that some modification of the $A_{\infty}$ category in 24 corresponds to the bounded derived category of coherent sheaves. However, in the case of two-tori which we shall discuss below, this problem is no matter.
} 
are regarded as open strings. Two open strings interact when their endpoints belong to the same D-branes just as the tensor product in the previous subsection. Correspondingly, a product structure $m_{2}$ is defined in the $A_{\infty}$-category. Essentially it is defined by summing up holomorphic disks 8 (see later arguments). It is then proved in [29] that the derived category of coherent sheaves and the $A_{\infty}$-category are equivalent as categories. This means, between these two categories, there exists a functor which induces both the isomorphism of objects and that of morphisms. In particular, the isomorphism between morphisms, i.e. the isomorphism between theta functions and the morphisms in the $A_{\infty^{-}}$ category, is compatible with the product structures on both sides. This implies that the product $m_{2}$ on the dual torus is compatible with the addition formula of theta functions on the torus with $\tau$ as mentioned in [24].

This is the correspondence of the two categories attending to $\tau=\hat{\rho}$. Of course, one can discuss the correspondence attending to $\rho=\hat{\tau}$ in the same way.

Next, we shall argue the relation of the above arguments to noncommutative tori. The argument above holds for general values of $\tau$, i.e. the two-tori with general flat backgrounds. Let us consider the Seiberg-Witten limit in this situation. It corresponds to the limit $\operatorname{Im} \rho \rightarrow 0$. Because $\rho=\hat{\tau}$, the complex structure of the dual torus is degenerate in this limit. Such tori are expressed in terms of noncommutative tori as argued previously. Namely, for such a degenerate two-torus one can define a noncommutative torus $U_{1} U_{2}=e^{-2 \pi i \frac{1}{\hat{\tau}}} U_{2} U_{1}$ where $-\frac{1}{\hat{\tau}}=-\frac{1}{B}=\theta$ ?. In this direction, the noncommutative compactification of module space of complex structures $\hat{\tau}$ for elliptic curves is discussed in [37, 38]. On the other hand, for the moduli parameter $\tau=\hat{\rho}$ the degeneration is irrelevant and the correspondence of the two categories explained above remains true in this limit. We shall, however, argue the situation related to the categories associated to $\tau=\hat{\rho}$ side below.

- A noncommutative homological mirror symmetry

As stated previously, projective modules are regarded as noncommutative analogue of vector bundles. Actually, for a Heisenberg module $E_{q, p, \theta}$ with $q-p \theta>0, p$ denotes the first Chern class of the projective module. $q$ then coincides with the rank of the projective module in the commutative case. Generally in noncommutative case, the rank is defined as $q-p \theta$, which is just identified with the value proportional to the mass of the $q$ D2- $-p$ D0 brane bound state[12, 13, 23].) Moreover, as stated in the previous subsection, the Heisenberg modules can also be regarded as bimodules. Note that when one regards Heisenberg modules $E_{q, p, \theta}$ and $E_{Q, P, \theta}$ as noncommutative vector bundles over

\footnotetext{
${ }^{8}$ Note that, however, the product structure does not depend on the holomorphic structure $\hat{\tau}=\rho$ and defined by using $\hat{\rho}=\tau$.

${ }^{9}$ The noncommutativity is actually $e^{-2 \pi i \frac{1}{\tilde{\tau}}}$ since we define the complex structure as in Eq.(16) and the noncommutative torus as in Subsection 3.1. We can also define the noncommutativity of the two-torus as $e^{2 \pi i \hat{\tau}}$ by changing these definitions relatively.
} 
$\mathcal{A}_{\theta}$, the $\mathcal{A}_{\theta^{\prime}}-\mathcal{A}_{\theta^{\prime \prime}}$ bimodule, which is also constructed as a Heisenberg module, is regarded as $\operatorname{Hom}\left(E_{q, p, \theta}, E_{Q, P, \theta}\right)$. Namely, these bimodules are regarded as noncommutative analogue of morphisms of the (derived) category of the coherent sheaves if a noncommutative analogue of the holomorphic structure is defined. On the other hand, in the previous subsection we construct the Heisenberg modules in the D1-brane picture. Since the D1-branes are lagrangian submanifolds, the Heisenberg modules are also expected to have a geometric picture of the $A_{\infty}$-category. Now on the noncommutative torus we consider an additional structure $\tau \in \mathbb{C}$ which is introduced as a noncommutative analogue of the holomorphic structure in [27] (for higher dimensional tori, see [26]). We shall then consider holomorphic submodules of the Heisenberg modules and show that the tensor product is equivalent to the $A_{\infty}$-structure $m_{2}$ on the dual torus (with background $\hat{E}$ ). Namely, the tensor product is realized as summing up the holomorphic disks. Since the definition of the $A_{\infty}$-category is complicated even for two-tori, we avoid to write down the definition. For the readers who would like to know the precise definition, see [29]. We shall first take the tensor product and then rewrite it to the form of $m_{2}$ of the $A_{\infty}$-category. Categorically, the result implies the compatibility of the product structures. The correspondence of objects and that of morphisms are more clear (compare the arguments below with[29]).

We have seen that the Heisenberg modules equip the constant curvature connection as in Eq.(13). It is known that all constant curvature connections on $E_{q, p, \theta}$ are given as

$$
\nabla_{1}=\frac{2 \pi i p}{q-p \theta} z+2 \pi i c_{1}, \quad \nabla_{2}=\frac{\partial}{\partial z}+2 \pi i c_{2}
$$

where $c_{1}, c_{2}$ are real numbers [17, 28]. Then consider the solution of the following equation

$$
\left(-\tau \nabla_{1}+\nabla_{2}\right) f(z, \mu)=0 .
$$

Its solutions are written in the form

$$
f(z, \mu)=a \exp \left(\pi i \tau \frac{p}{q-p \theta} z^{2}+2 \pi i\left(-\tau c_{1}+c_{2}\right) z\right), \quad a \in \mathbb{C}^{p} .
$$

$-\tau \nabla_{1}+\nabla_{2}$ is regarded as the noncommutative analogue of the Dolbeault operator $\bar{\partial}$, and the solutions are called holomorphic vectors 27]. Thus, for each Heisenberg module, one can consider its submodule over $\mathcal{A}_{\theta}$. The elements are expressed as linear combinations of the holomorphic vectors. The holomorphic vectors are labeled by the continuous parameter $-\tau c_{1}+c_{2} \in \mathbb{C}$, so the submodule is not so small. Moreover, the tensor product of two holomorphic vectors is also a holomorphic vector 27.

Now we express $a=a^{\mu} e_{\mu}$ where $e_{\mu}$ is the basis of the modules of $\mu$ and rewrite Eq.(17) in the following form

$$
f(z)=a^{\mu} e_{\mu} \exp \left(\pi i \tau \frac{p}{q-p \theta}\left(z-\frac{q-p \theta}{p} \epsilon\right)^{2}\right)
$$


where $\epsilon=c_{1}-c_{2} / \tau$. The tensor product is then written explicitly as

$$
\begin{aligned}
& \varphi\left(e_{\mu} \exp \left(\pi i \tau \frac{p}{q-p \theta}\left(z-\frac{q-p \theta}{p} \epsilon\right)^{2}\right) \otimes e_{\nu} \exp \left(\pi i \tau \frac{p^{\prime}}{q^{\prime}-p^{\prime} \theta^{\prime}}\left(z-\frac{q^{\prime}-p^{\prime} \theta^{\prime}}{p^{\prime}} \epsilon^{\prime}\right)^{2}\right)\right) \\
& =c_{\mu \nu}^{\rho} e_{\rho} \exp \left(\pi i \tau \frac{P}{Q-P \theta}\left(z-\frac{Q-P \theta}{P}\left(\epsilon+\frac{1}{q-p \theta} \epsilon^{\prime}\right)\right)^{2}\right), \\
& c_{\mu \nu}^{\rho}=\sum_{u \in \mathbb{Z}} \delta_{\mu}^{-r u+\rho} \delta_{\nu}^{u} \exp \left(\frac{\pi i \tau}{p p^{\prime} P}\left(P u-p^{\prime} \rho+p\left(q^{\prime}-p^{\prime} \theta^{\prime}\right) \epsilon^{\prime}-p^{\prime} \epsilon\right)^{2}\right) .
\end{aligned}
$$

Thus one can see that actually the holomorphic vectors close with respect to the tensor product. Moreover, this tensor product is associative. For $f \sim \exp \left(\pi i \tau \frac{p}{q-p \theta}\left(z-\frac{q-p \theta}{p} \epsilon\right)^{2}\right) \in$ $E_{q, p, \theta}, g \sim \exp \left(\pi i \tau \frac{p^{\prime}}{q^{\prime}-p^{\prime} \theta^{\prime}}\left(z-\frac{q^{\prime}-p^{\prime} \theta^{\prime}}{p^{\prime}} \epsilon^{\prime}\right)^{2}\right) \in E_{q^{\prime}, p^{\prime}, \theta^{\prime}}$ and $h \sim \exp \left(\pi i \tau \frac{p^{\prime \prime}}{q^{\prime \prime}-p^{\prime \prime} \theta^{\prime \prime}}\left(z-\frac{q^{\prime \prime}-p^{\prime \prime} \theta^{\prime \prime}}{p^{\prime \prime}} \epsilon^{\prime \prime}\right)^{2}\right) \in$ $E_{q^{\prime \prime}, p^{\prime \prime}, \theta^{\prime \prime}}, \varphi(f \otimes g \otimes h)$ becomes $\exp \left(\pi i \tau \frac{\mathcal{P}}{\mathcal{Q}-\mathcal{P} \theta}\left(z-\frac{\mathcal{Q}-\mathcal{P} \theta}{\mathcal{P}}\left(\epsilon+\frac{\epsilon^{\prime}}{q-p \theta}+\frac{\epsilon^{\prime \prime}}{Q-P \theta}\right)\right)^{2}\right)$ with an appropriate coefficient where

$$
\left(\begin{array}{ll}
R & P \\
S & Q
\end{array}\right)=\left(\begin{array}{ll}
r & p \\
s & q
\end{array}\right)\left(\begin{array}{ll}
r^{\prime} & p^{\prime} \\
s^{\prime} & q^{\prime}
\end{array}\right), \quad\left(\begin{array}{ll}
\mathcal{R} & \mathcal{P} \\
\mathcal{S} & \mathcal{Q}
\end{array}\right)=\left(\begin{array}{ll}
R & P \\
S & Q
\end{array}\right)\left(\begin{array}{ll}
r^{\prime \prime} & p^{\prime \prime} \\
s^{\prime \prime} & q^{\prime \prime}
\end{array}\right)
$$

We have a geometric realization about $\epsilon$ in holomorphic vectors and the structure constant $c_{\mu \nu}^{\rho}$ in Eq.(20). Such a realization will enable us to understand the relation between this tensor product and the $A_{\infty}$-category on two-tori. First, let us rewrite $\epsilon$ and $\epsilon^{\prime}$ as

$$
\epsilon:=\frac{q}{q-p \theta} \epsilon_{2}-\epsilon_{1}, \quad \epsilon^{\prime}:=(q-p \theta)\left(\frac{Q}{Q-P \theta} \epsilon_{3}-\frac{q}{q-p \theta} \epsilon_{2}\right) .
$$

The structure constant $c_{\mu \nu}^{\rho}$ is then expressed as

$$
c_{\mu \nu}^{\rho}:=\sum_{m \in \mathbb{Z}} \delta_{R\left(p^{\prime} m+Q \mu+q^{\prime} \nu\right)}^{\rho} \exp \left(2 \pi i \tau \triangle_{m}\right)
$$

where $\triangle_{m}$ is of the form

$$
\begin{aligned}
\triangle_{m} & =\frac{1}{2 p p^{\prime} P}\left(p p^{\prime} m+p q^{\prime} \nu-p^{\prime} \mu+p Q \epsilon_{3}+\epsilon_{1} p^{\prime}-P q \epsilon_{2}\right)^{2} \\
& =\frac{1}{2 p p^{\prime} P}\left(p p^{\prime} m+p q^{\prime} \nu-p^{\prime} \mu+p Q\left(\epsilon_{3}-\epsilon_{2}\right)-p^{\prime}\left(\epsilon_{2}-\epsilon_{1}\right)\right)^{2}
\end{aligned}
$$

Note that $\triangle_{m}$ for each $m, \mu, \nu$ and $\epsilon_{i}$ for $i=1,2,3$ is just the area of a triangle surrounded by three geodesic cycles on the covering space. For instance if $\epsilon_{i}=\alpha_{i}, \alpha_{i} \in \mathbb{R}$ for $i=1,2,3$ and $m=0$, then $\triangle_{0}$ coincides with the area of the triangle surrounded by three lines $y=-\alpha_{1}, y=\frac{p}{q} x-\alpha_{2}$ and $y=\frac{P}{Q} x-\alpha_{3}$ where $x:=x_{2}, y:=x_{1}$ (see Fig.6). Next, for simplicity we set $\epsilon_{i}=0$ and $\mu=\nu=0$. In this case $\triangle_{0}=0$ since the corresponding 


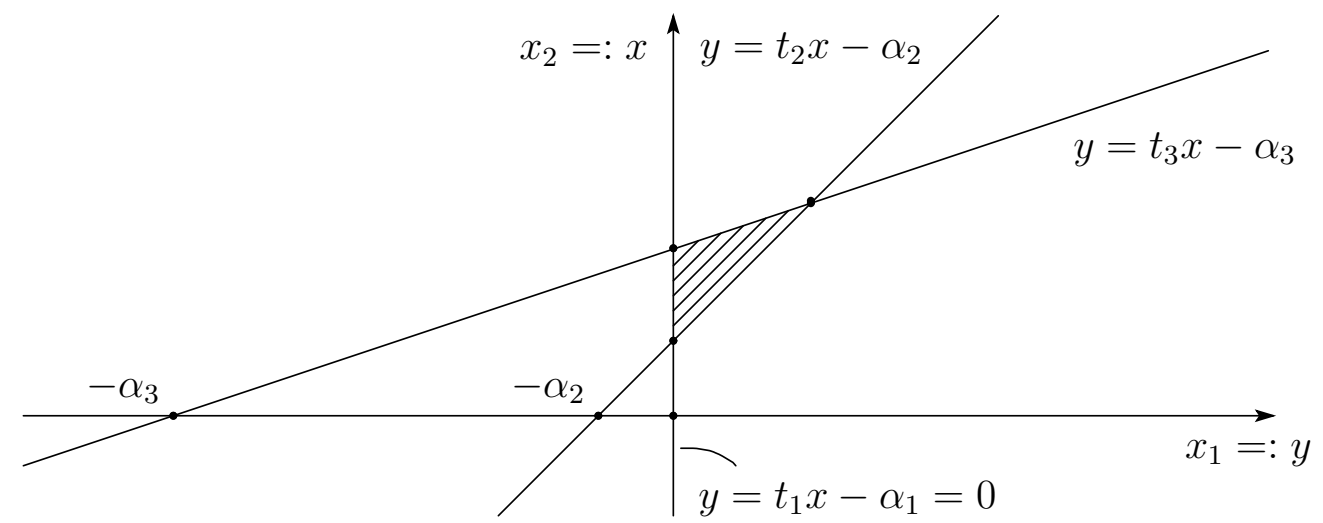

Figure 6: Three lines are the lagrangian submanifolds. We fix $\alpha_{1}=0$ by the translational invariance. For $t_{1}=0, t_{2}=\frac{p}{q}$ and $t_{3}=\frac{P}{Q}$, the area of the triangle surrounded by the three line is equal to the value $\triangle_{0}$ of $\mu=\nu=0$ and $\epsilon_{i}=\alpha_{i}$.

three lines intersect at the origin. $\triangle_{m}$ is then the area surrounded by $y=0, y=\frac{p}{q} x$ and $y-p m=\frac{P}{Q}(x-q m)$. The vertex $(x, y)=(0,0)$ corresponds to $\epsilon=\mu=0$. The vertex $(x, y)=(q m, p m)$ corresponds to $\epsilon^{\prime}=\nu=0$. While, the rest vertex $(x, y)=\left(0, \frac{p^{\prime} m}{P}\right)$ means that the vertex just corresponds to $\rho=p^{\prime} m$ up to $P$ (see Eq.(201). Fig.7] shows such a situation in the case $(p, q)=(1,1)$ and $(P, Q)=(3,1)\left(\left(p^{\prime}, q^{\prime}\right)=(2,1)\right)$. Note that the vertex $(x, y)=\left(0, \frac{p^{\prime} m}{P}\right)$ denotes the same point on the two-torus when $m$ is replaced to $m+P$. In this way, on two-tori there are infinitely many triangles whose vertices are common. The structure constant is then given by summing up all the triangles.

Generally (but in the case of $\left.t_{1}=\alpha_{1}=0\right), e_{\mu}, e_{\nu}$ and $e_{\rho}$ correspond to points $(x, y)=\left(\frac{q}{p}\left(\mu+\alpha_{1}\right), 0\right),(x, y)=\left(\frac{q^{\prime} q \nu+q Q\left(\alpha_{2}-\alpha_{3}\right)}{p^{\prime}}, \frac{q^{\prime} p \nu+q P \alpha_{2}-p Q \alpha_{3}}{p^{\prime}}\right)$ and $(x, y)=\left(\frac{Q}{P}\left(\rho+\alpha_{3}\right), 0\right)$, respectively, on the two-torus. Since the slope of the three lines $t_{i}$ are given, the triangle is obtained when the two points $e_{\mu}$ and $e_{\nu}$ are determined. The condition that the rest third vertex coincides with $e_{\rho}$ is then the $\delta_{R\left(p^{\prime} m+Q \mu+q^{\prime} \nu\right)}^{\rho}$ in Eq.(21). One can see that the place of these lines depend not only on $\alpha_{i}$ but on $\mu$ and $\nu$. For instance for the holomorphic vector which belongs to the $\mathcal{A}_{\theta^{-}} \mathcal{A}_{\theta^{\prime}}$ bimodule, the line of $i=2$ is described by $y=\frac{p}{q}\left(x-\frac{q \mu}{p}\right)-\alpha_{2}$. This agrees with the viewpoints of the Heisenberg module in the previous subsection. In fact, we have identified $f(z, \mu)$ as the function on the line $y=\frac{p}{q}\left(x-\frac{q \mu}{p}\right)$, which just coincides with the situation here This fact holds in a similar way for any holomorphic vector defined between any two D1-brane, since the arguments can reduce to the above ones by a $S L(2, \mathbb{Z})$ transformation.

Let us denote $\epsilon_{i}=\alpha_{i}+\frac{\beta_{i}}{\tau}$ where $\alpha_{i}, \beta_{i} \in \mathbb{R}$. As explained above, $\alpha_{i}$ expresses the place of the D1-brane $i$. Then what is $\beta_{i}$ ? By comparing the holomorphic vector in Eq.(17) and Eq.(18), we can see that it corresponds to $c_{2}$. (We can also see that $\alpha_{i}$ corresponds to

\footnotetext{
${ }^{10} \mathrm{In}$ fact, in the previous subsection one could use other identifications which differ by the translation $\alpha_{i}$.
} 


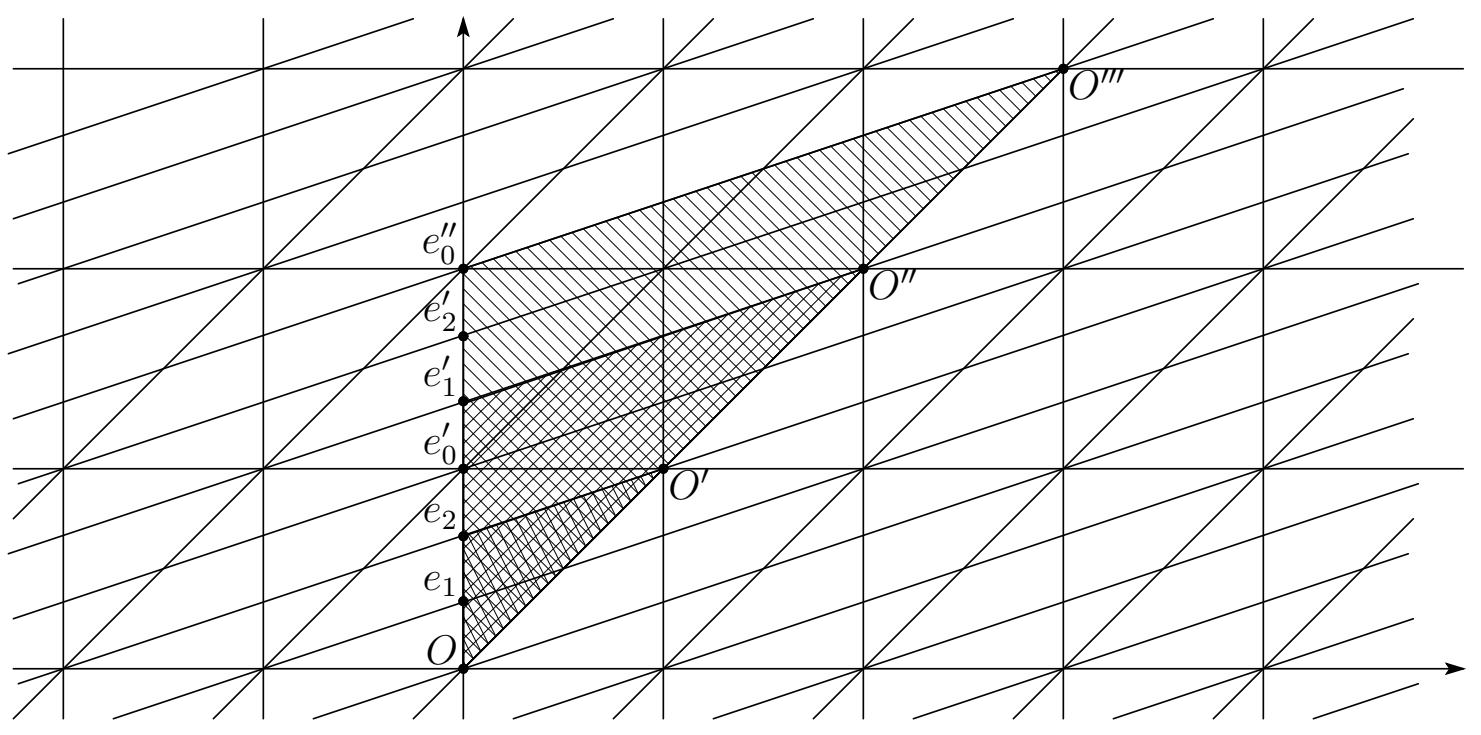

Figure 7: The area of the triangle times the complexified symplectic form gives the coefficient of the tensor product. This is the figure in the case $(p, q)=(1,1),(P, Q)=(3,1)$ $\left(\left(p^{\prime}, q^{\prime}\right)=(2,1)\right), \epsilon_{i}=0$ and $\nu=0$ (since $p=1, \mu=0$ originally). $e_{\mu=0}, e_{\nu=0}$ and $e_{\rho=0}$ correspond to the point $O .{ }^{\prime}$ means the mirror points (in the sense of the covering) of the original point. $e_{i} \mathrm{~s}$ in this picture express $e_{\rho=i}$. The triangle $O O^{\prime} e_{2}, O O^{\prime \prime} e_{1}^{\prime}$ and $O O^{\prime \prime \prime} e_{0}^{\prime \prime}$ correspond to $\triangle_{m=1}, \triangle_{m=2}$ and $\triangle_{m=3}$, respectively.

$c_{1}$.) Furthermore it means that we consider the holomorphic vector with $\nabla_{2}=\frac{\partial}{\partial z}+2 \pi i c_{2}$. This is nothing but the holonomy on the D1-brane parameterized by $z$. More precisely, $\beta_{i}$ corresponds to the holonomy on the corresponding D1-brane for each $i$. For instance, for the holomorphic vector which belongs to the $\mathcal{A}_{\theta^{\prime}}-\mathcal{A}_{\theta^{\prime \prime}}$ module, the connection $\nabla_{2}$ is $\nabla_{2}=\frac{\partial}{\partial z}-2 \pi i(q-p \theta)\left(\frac{Q}{Q-P \theta} \beta_{3}-\frac{q}{q-p \theta} \beta_{2}\right)$. Here the constant term proportional to $\beta_{3}$ is regarded as an element in the center of $\mathcal{A}_{\theta^{\prime \prime}}$, whereas that proportional to $\beta_{2}$ is realized as a constant in $\mathcal{A}_{\theta^{\prime}}$. The scaling $\frac{Q}{Q-P \theta}$ and $\frac{q}{q-p \theta}$ in front of $\beta_{i}$ s are also consistent with the identification of the coordinate $z$ in Fig. $\Theta$. Then, the term linear for $\beta_{i} \mathrm{~s}$ in $\triangle_{m}$ coincides with the integral of the flat connection along the boundary of the triangle, i.e. the three D1-branes. Note that the triangle is an open string disk, so the integral is equal to that of flat gauge field along the boundary of the disk. These contributions of $\alpha_{i}$ and $\beta_{i}$ to the structure constant $c_{\mu \nu}^{\rho}$ are just the definition of the $A_{\infty}$-category in [39, 24]. The terms of $\beta_{i}$ square in $\triangle_{m}$ can be absorbed into the definition of the expression of the holomorphic vectors, and one can see the correspondence of the arguments here with those in [29].

We have taken the tensor product between the 'holomorphic' $\mathcal{A}_{\theta^{-}} \mathcal{A}_{\theta^{\prime}}$ bimodule and $\mathcal{A}_{\theta^{\prime}}-\mathcal{A}_{\theta^{\prime \prime}}$ bimodule and obtained the $\mathcal{A}_{\theta^{-}} \mathcal{A}_{\theta^{\prime \prime}}$ bimodule. From the viewpoints of the $A_{\infty}-$ category in [29], the tensor product corresponds to the product $m_{2}$ between the morphisms in the following situation; both morphisms are the homomorphisms between flat line 
bundles on lagrangian submanifolds, and one of these three lagrangian submanifolds is fixed to be $y=0$ (or $y=-\alpha_{1}$ ). In order to get the category of the holomorphic vectors which corresponds to the $A_{\infty}$-category of [29], it is sufficient to consider the tensor product in a more general case, the tensor product between $\operatorname{Mat}_{n_{1}}\left(\mathcal{A}_{\theta^{\prime}}\right)-\operatorname{Mat}_{n_{2}}\left(\mathcal{A}_{\theta^{\prime \prime}}\right)$ bimodule and $\operatorname{Mat}_{n_{2}}\left(\mathcal{A}_{\theta^{\prime \prime}}\right)-\operatorname{Mat}_{n_{3}}\left(\mathcal{A}_{\theta^{\prime \prime \prime}}\right)$ bimodule. Here $\operatorname{Mat}_{n}\left(\mathcal{A}_{\theta}\right)$ denotes $n \times n$ matrix with $\mathcal{A}_{\theta}$ entry, which is the endomorphism algebra of the rank $n$ free module over $\mathcal{A}_{\theta}$. First, one can easily define the tensor product between an $\mathcal{A}_{\theta^{\prime}}-\mathcal{A}_{\theta^{\prime \prime}}$ module and an $\mathcal{A}_{\theta^{\prime \prime}}-\mathcal{A}_{\theta^{\prime \prime \prime}}$ module over $\mathcal{A}_{\theta}$ by regarding it as the tensor product over the noncommutative torus $\mathcal{A}_{\theta^{\prime}}$ and using Eq.(14). The structure constant is then determined by summing up the area of the triangles as in Fig.6 where $t_{1} \in \mathbb{Q}$. Next, to extend it further for $\operatorname{Mat}_{n_{i}}\left(\mathcal{A}_{\theta^{\prime}}\right)-\operatorname{Mat}_{n_{i+1}}\left(\mathcal{A}_{\theta^{\prime \prime}}\right)$ bimodules, essentially one may multiply $V_{i} \otimes V_{i+1}^{*}$ to holomorphic vectors in the $\mathcal{A}_{\theta^{\prime}}-\mathcal{A}_{\theta^{\prime \prime}}$ bimodule, where $V_{i}=\mathbb{C}^{n_{i}}$. However, the space of morphisms of the $A_{\infty}$-category in [29] is larger. By comparing our arguments with those in [29], one can see how the space of the holomorphic vectors should be extended to recover any morphism of the $A_{\infty}$-category in [29]. The connection $\nabla_{2}$ should be extended by replacing $\beta_{i}$ to $\beta_{i}+N_{i}$ where $N_{i} \in \operatorname{End} V_{i}$ is a constant indecomposable nilpotent matrix. We keep $\nabla_{1}$ unchanged. Therefore the curvature $\left[\nabla_{1}, \nabla_{2}\right]=\frac{2 \pi i p^{\prime}}{q^{\prime}-p^{\prime} \theta}$ is preserved through this modification. Then we may consider, as the extended holomorphic vectors, the solutions of the equation $\left(-\tau \nabla_{1}+\nabla_{2}\right) f=0$ where $f$ belongs to the $\operatorname{Mat}_{n_{i}}\left(\mathcal{A}_{\theta^{\prime}}\right)-\operatorname{Mat}_{n_{i+1}}\left(\mathcal{A}_{\theta^{\prime \prime}}\right)$ bimodule.

In [29], the explicit form of $m_{2}$ is given in the case $p=p^{\prime}=1$ so in this case one can immediately confirm that Eq.(20) or Eq.(22) actually coincides with the result in [29]. The associativity of the tensor product can be checked directly, but it is also realized geometrically as the way of separating each quadrangle into two triangles [39, 40].

To summarize, the $A_{\infty}$-category is defined by using lagrangian submanifolds, that is, the D1-branes. Similarly, the Heisenberg modules are also defined by the D1-branes. In this picture, $m_{2}$ in the $A_{\infty}$-structure is identified with the tensor product between bimodules. Namely, the open string interactions on noncommutative two-tori are given by counting holomorphic disks i.e. disk instantons on two-tori.

- Additional comments

Indeed we have seen that the category of holomorphic vectors corresponds to the $A_{\infty}$-category for general $\theta$. However note that the expression of the holomorphic vectors depends on $\theta$. As seen from the expression in Eq.(17), holomorphic vectors exist only if $\frac{p}{q-p \theta}>0$. Morphisms of the $A_{\infty}$-category are then in one-to-one correspondence with the holomorphic vectors only if $\theta=0$. Thus the category of the holomorphic vectors depends on $\theta$. In this sense, we can say that the category of the holomorphic vectors gives a noncommutative extension of the $A_{\infty}$-category (when higher products $m_{i}$ for $i \geq 3$ are also constructed). Moreover, the morphisms of the $A_{\infty}$-category correspond to theta functions which are the morphisms of the (derived) category of coherent sheaves. Therefore these holomorphic vectors can be regarded as certain noncommutative theta 
functions. In [26] on higher dimensional tori the holomorphic vectors are discussed from the point of view of noncommutative analogue of theta functions. For other literatures about noncommutative extensions of theta functions, see 41, 42].

As stated previously, the $A_{\infty}$-category possesses higher product $m_{i}$ for $i \geq 3$ [40]. For lagrangian submanifolds on the covering space of the two-tori, the higher products $m_{i}$ are constructed by summing over the $i+1$-gons in a similar way as $m_{2}$. The corresponding higher products of the holomorphic vectors can also be constructed, since the graphical realization of the holomorphic vectors is given in this paper. The relations of the $A_{\infty}$ structure are also realized as ways of separating polygons into two polygons [39, 40]. It is shown that, on the derived category of coherent sheaves side, the higher product of the $A_{\infty}$-structure correspond to the higher Massey products [39, 40]. Therefore, the higher products of the holomorphic vectors may be regarded as noncommutative higher Massey products.

Finally, we mention about a symmetry that the category of the holomorphic vectors has. In the commutative case $\theta=0, \epsilon_{i}$ s have a translational invariance. This is a property which the morphisms of the $A_{\infty}$-category have. For $\theta \neq 0$ the structure constant (20) still has the translational invariance, but each holomorphic vector does not. This means, in other words, that the holomorphic vectors have a symmetry corresponding to the translational invariance of $\epsilon_{i}$. When $\theta=0$ this symmetry becomes degenerate.

\section{Conclusions and Discussions}

In this paper, we related the physics of the D1-brane on two-tori to the Kronecker foliation. The product of the field corresponding to the open strings ending on the D1-brane was identified with the crossed product of the rotation algebras. The algebra of the fields was then identified with the noncommutative two-tori represented by the rotation algebra. We showed that, in a particular degenerate metric, the two-torus is 'foliated'. That is, when one probes the physics on the two-torus by a D1-brane on a 1-cycle, the two-torus is 'foliated' by the open strings ending on the D1-brane. Such a situation is described by the the Kronecker foliation, so the different choice of the 1-cycle on which the probe D1brane lies are related by the Morita equivalence of the noncommutative two-tori. Thus the Morita equivalence of noncommutative two-tori was realized from the D1-brane physics geometrically. The degenerate metric is T-dual to the metric in the Seiberg-Witten limit. It is known that the open string theory in the Seiberg-Witten limit is realized from the viewpoints of the deformation quantization. Therefore, we can conclude that the two representations of noncommutative two-tori, that in the deformation quantization and that in the crossed product, are related by T-duality. Such viewpoints are then applied to the homological mirror symmetry. After the tensor product between two Heisenberg modules is constructed, the tensor product between two holomorphic vectors is calculated 
and it is identified with the $m_{2}$ in the $A_{\infty}$-structure defined in [29]. This implies that the interaction of two 'holomorphic' open strings between noncommutative D-branes on noncommutative two-torus can be obtained from disk instanton contributions on twotori. This results can be regarded as a 'noncommutative homological mirror symmetry' on two-tori.

Though we discussed the arguments above on the special degenerate metric, one might generalize it in the case of any nondegenerate metric by introducing additional moduli $\Phi[43$ as in 22]. For higher dimensional tori, the noncommutative expression of the open string physics is essentially obtained by quantizing open strings in general boundary conditions 23]. However, it is expected that the homological mirror symmetry cannot understood by straightforward extensions of the two-dimensional case [39, 44]. Therefore, even if the tensor product of projective modules is constructed, it is unclear that the category of the holomorphic vectors is related completely to the homological mirror symmetry. Conversely, the construction of the tensor product in higher dimensional tori might give an insight for the homological mirror symmetry for higher dimensional tori.

Different connections between noncommutative tori and the homological mirror symmetry are found in 44, 37, 38, 45, 44, for example. In 46] lagrangian foliations are considered as the objects of the $A_{\infty}$-category. In [37, 38 in the degenerate limit of the complex structure its noncommutative compactification is discussed.

Formally, if one considers the direct sum of all objects of an $A_{\infty}$-category, the morphisms are unified as the endomorphism algebra which acts on it. In such a way, formally an $A_{\infty}$-category can be regarded as an $A_{\infty}$-algebra of the endomorphism algebra (for the definition of $A_{\infty}$-algebras and a deep relation of them to the field theory of open string, see 47]). Alternatively, by definition, any projective module over $\mathcal{A}_{\theta}$ is obtained by multiplying an appropriate idemponent on free module $\left(\mathcal{A}_{\theta}\right)^{N}$ for sufficiently large $N$. Bimodules are also obtained by multiplying appropriate idemponents on $\operatorname{Mat}_{N}\left(\mathcal{A}_{\theta}\right)$ from left and right. This implies that the $A_{\infty}$-category is naturally embedded into the $A_{\infty}$-algebra of $\operatorname{Mat}_{N}\left(\mathcal{A}_{\theta}\right)$. Thus, physically, the results of this paper might be relevant to nonperturbative effects of large $N$ field theory over $\mathcal{A}_{\theta}$ through tachyon condensation process.

\section{Acknowledgments}

I am very grateful to A. Kato for helpful discussions and advice. I would also like to thank Y. Terashima for valuable discussions. The author is supported by JSPS Research Fellowships for Young Scientists.

\section{References}


[1] A. Connes, M. R. Douglas and A. Schwarz, "Noncommutative geometry and matrix theory: Compactification on tori," JHEP 9802 (1998) 003, hep-th/9711162.

[2] M. R. Douglas and C. Hull, "D-branes and the noncommutative torus," JHEP 9802 (1998) 008, hep-th/9711165.

[3] T. Kawano and K. Okuyama, "Matrix theory on noncommutative torus," Phys. Lett. B 433 (1998) 29, hep-th/9803044.

[4] Y. E. Cheung and M. Krogh, "Noncommutative geometry from 0-branes in a background B-field," Nucl. Phys. B 528 (1998) 185, hep-th/9803031.

[5] V. Schomerus, "D-branes and deformation quantization," JHEP 9906 (1999) 030 arXiv:hep-th/9903205.

[6] N. Seiberg and E. Witten, "String theory and noncommutative geometry," JHEP 9909 (1999) 032, hep-th/9908142.

[7] A. S. Cattaneo and G. Felder, "A path integral approach to the Kontsevich quantization formula," Commun. Math. Phys. 212 (2000) 591 arXiv:math.qa/9902090.

[8] M. Rieffel, " $C^{*}$-algebras associated with irrational rotations," Pacific J. Math. 93(2)(1981)415-429.

[9] M. Rieffel, "Projective modules over higher-dimensional non-commutative tori," Canadian J. Math. 40(1988), 257-338.

[10] M. Rieffel and A. Schwarz, "Morita equivalence of multidimensional noncommutative tori," Internat. J. Math.10 (2) (1999) 289-299, math.QA/9803057.

[11] A. Schwarz, "Morita equivalence and duality," Nucl. Phys. B 534 (1998) 720, hepth/9805034.

[12] I. Bars, H. Kajiura, Y. Matsuo and T. Takayanagi, "Tachyon condensation on noncommutative torus," Phys. Rev. D 63 (2001) 086001, hep-th/0010101.

[13] H. Kajiura, Y. Matsuo and T. Takayanagi, "Exact tachyon condensation on noncommutative torus," hep-th/0104143.

[14] A. Sen, "Tachyon condensation on the brane antibrane system," JHEP 9808 (1998) 012 hep-th/9805170. "Non-BPS states and branes in string theory," hep-th/9904207.

[15] F. Bayen, M. Flato, C. Frønsdal, A. Lichnerowicz, D. Sternheimer, "Deformation theory and quantization I, II," Ann. Phys. 111 (1978), 61-110, 111-151. 
[16] A. Connes, "Noncommutative geometry," Academic Press 1994.

[17] A. Connes and M. Rieffel, "Yang-Mills for non-commutative two-tori," Contemp. Math. 62(1987) 237-265.

[18] P. Ho, "Twisted bundle on quantum torus and BPS states in matrix theory," Phys. Lett. B 434 (1998) 41, hep-th/9803166.

[19] B. Morariu and B. Zumino, "Super Yang-Mills on the noncommutative torus," hepth/9807198.

[20] D. Brace, B. Morariu and B. Zumino, "Dualities of the matrix model from T-duality of the type II string," Nucl. Phys. B 545 (1999) 192, hep-th/9810099.

[21] F. Ardalan, H. Arfaei and M. M. Sheikh-Jabbari, "Noncommutative geometry from strings and branes," JHEP 9902 (1999) 016, hep-th/9810072.

[22] Y. Imamura, "T-duality of non-commutative gauge theories," JHEP 0001 (2000) 039 arXiv:hep-th/0001105.

[23] H. Kajiura, "T-duality group for open string theory," arXiv:hep-th/0105056.

[24] M. Kontsevich, "Homological algebra of mirror symmetry," Proceedings of the International Congress of Mathematicians, Vol. 1, 2 (Zürich, 1994), 120-139, Birkhäuser, Basel, 1995.

[25] E. Witten, "Chern-Simons gauge theory as a string theory," hep-th/9207094.

[26] A. Schwarz, "Theta functions on noncommutative tori," Lett. Math. Phys. 58 (2001), no. 1, 81-90. math.QA/0107186.

[27] M. Dieng and A. Schwarz, "Differential and complex geometry of two-dimensional noncommutative tori," math.QA/0203160.

[28] A. Konechny and A. Schwarz, "Introduction to M(atrix) theory and noncommutative geometry," hep-th/0012145, and references therein.

[29] A. Polishchuk and E. Zaslow, "Categorical mirror symmetry: the elliptic curve," Adv. Theor. Math. Phys. 2 (1998), no. 2, 443-470. math.AG/9801119.

[30] S. I. Gelfand and Y. I. Manin, "Homological Algebra," Springer-Verlag, Berlin, Heidelberg, 1994.

[31] J. Distler, H. Jockers and H. Park, arXiv:hep-th/0206242. 
[32] M. R. Douglas, "D-branes, categories and N = 1 supersymmetry," J.Math.Phys. 42 (2001) 2818-2843, arXiv:hep-th/0011017. "D-branes and N = 1 supersymmetry," arXiv:hep-th/0105014.

[33] P. S. Aspinwall and A. E. Lawrence, "Derived categories and zero-brane stability," JHEP 0108 (2001) 004 arXiv:hep-th/0104147.

[34] D. E. Diaconescu, "Enhanced D-brane categories from string field theory," JHEP 0106 (2001) 016 arXiv:hep-th/0104200.

[35] K. Fukaya, "Morse homotopy, $A^{\infty}$-category, and Floer homologies," Proceedings of GARC Workshop on Geometry and Topology '93 (Seoul, 1993), 1-102, Lecture Notes Ser., 18, Seoul Nat. Univ., Seoul, 1993.

[36] R. Dijkgraaf, E. Verlinde and H. Verlinde, "On Moduli Spaces Of Conformal Field Theories With $C \geq 1$," IN *COPENHAGEN 1987, PROCEEDINGS, PERSPECTIVES IN STRING THEORY* 11\%-13\%. . "Conformal Field Theory At C = 1," THU-87/27 Talk presented at the 1987 Cargese Summer School on Nonperturbative Quantum Field Theory, Cargese, Italy, Jul 16 - 30, 198\%. "C = 1 Conformal Field Theories On Riemann Surfaces," Commun. Math. Phys. 115 (1988) 649.

[37] Y. Soibelman, "Quantum tori, mirror symmetry and deformation theory," Lett. Math. Phys. 56 (2001) 99 arXiv:math.qa/0011162.

[38] Y. Soibelman and V. Vologodsky, "Non-commutative compactifications and elliptic curves," arXiv:math.ag/0205117.

[39] K. Fukaya, "Mirror symmetry of abelian varieties and multi theta functions," preprint Kyoto University, 1998.

[40] A. Polishchuk, " $A_{\infty}$-structures on an elliptic curve," math.AG/0001048. "Homological mirror symmetry with higher products," math.AG/9901025.

[41] "A. Weinstein, Classical theta functions and quantum tori," Publ. RIMS. Kyoto Univ., vol. 30(1994), p. 327-333.

[42] Y. Manin, "Quantized theta-function," Preprint RIMS, RIMS-700, 1990. "Mirror symmetry and quantization of abelian varieties," math.AG/0005143.

[43] B. Pioline and A. Schwarz, "Morita equivalence and T-duality (or B versus $\Theta$ )," JHEP 9908 (1999) 021, hep-th/9908019.

[44] A. Kapustin and D. Orlov, "Vertex algebras, mirror symmetry, and D-branes: The case of complex tori," arXiv:hep-th/0010293. 
[45] P. Bressler and Y. Soibelman, "Mirror symmetry and deformation quantization," arXiv:hep-th/0202128.

[46] K. Fukaya, "Floer homology of Lagrangian foliation and non-commutative mirror symmetry," Preprint 98-08, Kyoto University, 1998.

[47] H. Kajiura, "Homotopy algebra morphism and geometry of classical string field theories," Nucl. Phys. B 630 (2002) 361 arXiv:hep-th/0112228. 